\title{
Binary Biometric Representation through Pairwise Adaptive Phase Quantization
}

\author{
Chun Chen and Raymond Veldhuis \\ Department of Electrical Engineering Mathematics and Computer Science, University of Twente, 7500 AE Enschede, The Netherlands \\ Correspondence should be addressed to Chun Chen, c.chen@nki.nl
}

Received 18 October 2010; Accepted 24 January 2011

Academic Editor: Bernadette Dorizzi

Copyright (๑) 2011 C. Chen and R. Veldhuis. This is an open access article distributed under the Creative Commons Attribution License, which permits unrestricted use, distribution, and reproduction in any medium, provided the original work is properly cited.

\begin{abstract}
Extracting binary strings from real-valued biometric templates is a fundamental step in template compression and protection systems, such as fuzzy commitment, fuzzy extractor, secure sketch, and helper data systems. Quantization and coding is the straightforward way to extract binary representations from arbitrary real-valued biometric modalities. In this paper, we propose a pairwise adaptive phase quantization (APQ) method, together with a long-short (LS) pairing strategy, which aims to maximize the overall detection rate. Experimental results on the FVC2000 fingerprint and the FRGC face database show reasonably good verification performances.
\end{abstract}

\section{Introduction}

Extracting binary biometric strings is a fundamental step in template compression and protection [1]. It is well known that biometric information is unique, yet inevitably noisy, leading to intraclass variations. Therefore, the binary strings are desired not only to be discriminative, but also have to low intraclass variations. Such requirements translate to both low false acceptance rate (FAR) and low false rejection rate (FRR). Additionally, from the template protection perspective, we know that general biometric information is always public, thus any person has some knowledge of the distribution of biometric features. Furthermore, the biometric bits in the binary string should be independent and identically distributed (i.i.d.), in order to maximize the attacker's efforts in guessing the target template.

Several biometric template protection concepts have been published. Cancelable biometrics $[2,3]$ distort the image of a face or a fingerprint by using a one-way geometric distortion function. The fuzzy vault method $[4,5]$ is a cryptographic construction allowing to store a secret in a vault that can be locked using a possibly unordered set of features, for example, fingerprint minutiae. A third group of techniques, containing fuzzy commitment [6], fuzzy extractor [7], secure sketch [8], and helper data system [9-
13], derive a binary string from a biometric measurement and store an irreversibly hashed version of the string with or without binding a crypto key. In this paper, we adopt the third group of techniques.

The straightforward way to extract binary strings is quantization and coding of the real-valued features. So far, many works [9-11, 14-20] have adopted the bit extraction framework shown in Figure 1, involving two tasks: (1) designing a one-dimensional quantizer and (2) determining the number of quantization bits for every feature. The final binary string is then the concatenation of the output bits from all the individual features.

Designing a one-dimensional quantizer relies on two probability density functions (PDFs): the background PDF and the genuine user PDF, representing the probability density of the entire population and the genuine user, respectively. Based on the two PDFs, quantization intervals are determined to maximize the detection rate, subject to a given FAR, according to the Neyman-Pearson criterion. So far, a number of one-dimensional quantizers have been proposed [9-11, 14-17], as categorized in Table 1. Quantizers in [9-11] are userindependent, constructed merely from the background PDF, whereas quantizers in [14-17] are userspecific, constructed from both the genuine user PDF and the background PDF. Theoretically, user-specific quantizers 


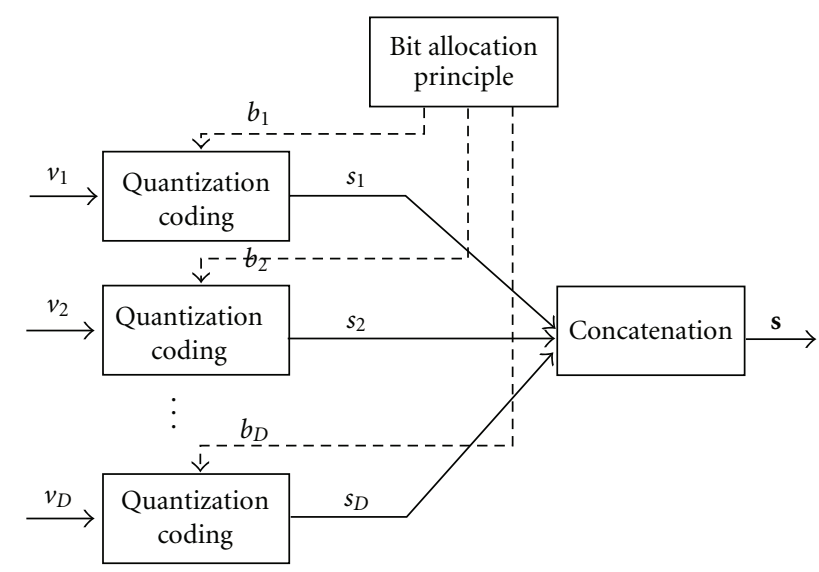

Figure 1: The bit extraction framework based on the onedimensional quantization and coding, where $D$ denotes the number of features; $b_{i}$ denotes the number of quantization bits for the $i$ th feature $(i=1, \ldots, D)$, and $s_{i}$ denotes the output bits. The final binary string is $\mathbf{s}=s_{1} s_{2} \cdots s_{D}$.

provide better verification performances. Particularly, the likelihood ratio-based quantizer [17], among all the quantizers, is optimal in the Neyman-Pearson sense. Quantizers in $[9,14-16]$ have equal-width intervals. Unfortunately, this leads to potential threats: features obtain higher probabilities in certain quantization intervals than in others, and thus attackers can easily find the genuine interval by continuously guessing the one with the highest probability. To avoid this problem, quantizers in $[10,11,17]$ have equal-probability intervals, ensuring i.i.d. bits.

Apart from the one-dimensional quantizer design, some papers focus on assigning a varying number of quantization bits to each feature. So far, several bit allocation principles have been proposed: fixed bit allocation (FBA) $[10,11,17]$ simply assigns a fixed number of bits to each feature. On the contrary, the detection rate optimized bit allocation (DROBA) [19] and the area under the FRR curve optimized bit allocation (AUF-OBA) [20], assign a variable number of bits to each feature, according to the features' distinctiveness. Generally, AUF-OBA and DROBA outperform FBA.

In this paper, we deal with quantizer design rather than assigning the quantization bits to features. Although onedimensional quantizers yield reasonably good performances, a problem remains: independency between all feature dimensions is usually difficult to achieve. Furthermore, onedimensional quantization leads to inflexible quantization intervals, for instance, the orthogonal boundaries in the two-dimensional feature space, as illustrated in Figure 2(a). Contrarily, two-dimensional quantizers, with an extra degree of freedom, bring more flexible quantizer structures. Therefore, a user-independent pairwise polar quantization was proposed in [21]. The polar quantizer is illustrated in Figure 2(b), where both the magnitude and the phase intervals are determined merely by the background PDF. In principle, polar quantization is less prone to outliers and less strict on independency of the features, when the genuine user PDF is located far from the origin. Therefore, in [21], two
TABLE 1: The categorized one-dimensional quantizers.

\begin{tabular}{ll}
\hline User independent & User specific \\
\hline Linnartz and Tuyls [9] & Vielhauer et al. [14] \\
Tuyls et al. [10] & Feng and Wah [15] \\
Kevenaar et al. [11] & Chang et al. [16] \\
& Chen et al. [17] \\
\hline Equal width & Equal probability \\
\hline Linnartz and Tuyls [9] & Tuyls et al. [10] \\
Vielhauer et al. [14] & Kevenaar et al. [11] \\
Feng and Wah [15] & Chen et al. [17] \\
Chang et al. [16] & \\
\hline
\end{tabular}

pairing strategies, the long-long and the long-short pairing, were proposed for the magnitude and the phase, respectively. Both pairing strategies use the Euclidean distances between each feature's mean and the origin. Results showed that the magnitude yields a poor verification performance, whereas the phase yields a good performance. The two-dimensional quantization-based bit extraction framework, including an extra feature pairing step, is illustrated in Figure 3.

Since the phase quantization has shown in [21] to yield a good performance, in this paper, we propose a userspecific adaptive phase quantizer (APQ). Furthermore, we introduce a Mahalanobis distance-based long-short (LS) pairing strategy that by good approximation maximizes the theoretical overall detection rate at zero Hamming distance threshold.

In Section 2 we introduce the adaptive phase quantizer (APQ), with simulations in a particular case with independent Gaussian densities. In Section 3 the long-short (LS) pairing strategy is introduced to compose pairwise features. In Section 4, we give some experimental results on the FVC2000 fingerprint database and the FRGC face database. In Section 5 the results are discussed and conclusions are drawn in Section 6.

\section{Adaptive Phase Quantizer (APQ)}

In this section, we first introduce the APQ. Afterwards, we discuss its performance in a particular case where the feature pairs have independent Gaussian densities.

2.1. Adaptive Phase Quantizer (APQ). The adaptive phase quantization can be applied to a two-dimensional feature vector if its background PDF is circularly symmetric about the origin. Let $\mathbf{v}=\left\{v_{1}, v_{2}\right\}$ denote a two-dimensional feature vector. The phase $\theta=\operatorname{angle}\left(v_{1}, v_{2}\right)$, ranging from $[0,2 \pi)$, is defined as its counterclockwise angle from the $v_{1}$-axis. For a genuine user $\omega$, a $b$-bit APQ is then constructed as

$$
\begin{aligned}
\xi & =\frac{2 \pi}{2^{b}}, \\
Q_{\omega, j} & =\left(\varphi_{\omega}^{*}+(j-1) \xi \bmod 2 \pi, \varphi_{\omega}^{*}+j \xi \bmod 2 \pi\right], \\
& j=1, \ldots, 2^{b},
\end{aligned}
$$




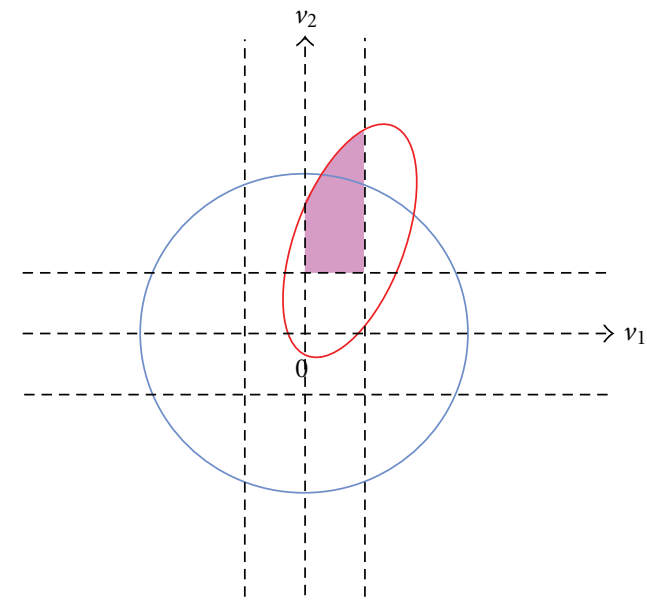

(a)

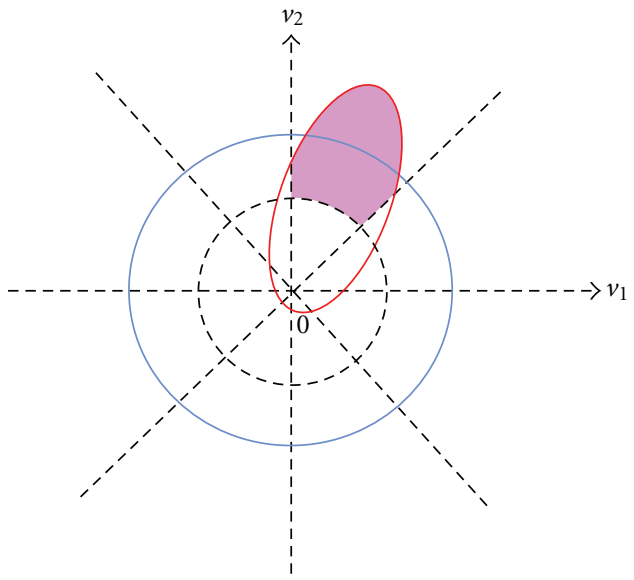

(b)

FIGURE 2: The two-dimensional illustration of (a) the one-dimensional quantizer boundaries (dash line) and (b) the userindependent polar quantization boundaries (dash line). The genuine user PDF is in red and the background PDF is in blue. The detection rate and the FAR are the integral of both PDFs in the pink area.

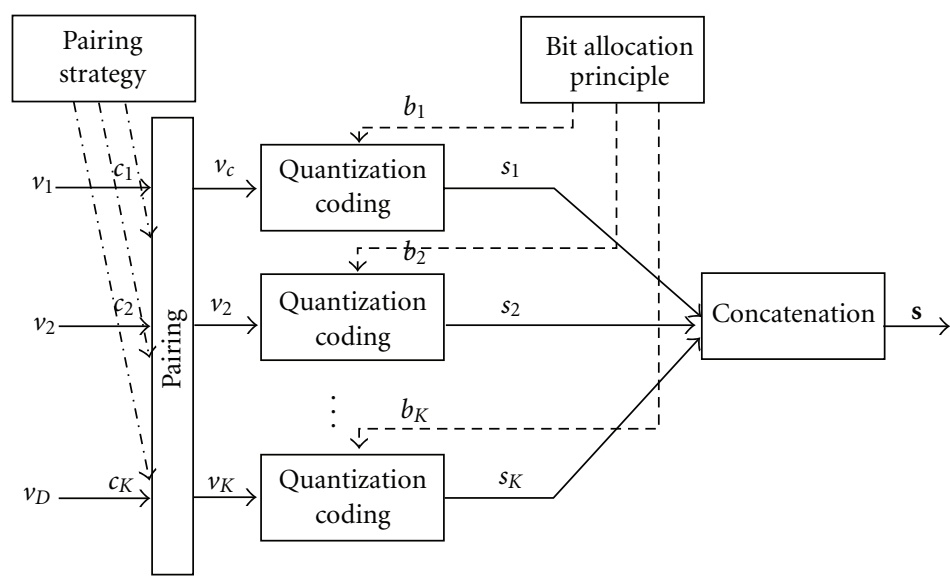

Figure 3: The bits extraction framework based on two-dimensional quantization and coding, where $D$ denotes the number of features; $K$ denotes the number of feature pairs; $c_{k}$ denotes the feature index for the $k$ th feature pair $(k=1, \ldots, K) ; s_{i}$ denotes the corresponding quantized bits. The final output binary string is $S=s_{1} s_{2} \cdots s_{K}$.

where $Q_{\omega, j}$ represents the $j$ th quantization interval, determined by the quantization step $\xi$ and an offset angle $\varphi_{\omega}^{*}$. Every quantization interval is uniquely encoded using $b$ bits. Let $\boldsymbol{\mu}_{\omega}$ be the mean of the genuine feature vector $\mathbf{v}$, then among the intervals, the genuine interval $Q_{\omega \text {,genuine, which is }}$ assigned for the genuine user $\omega$, is referred to as

$$
Q_{\omega, j}=Q_{\omega, \text { genuine }} \Leftrightarrow \mu_{\omega} \in Q_{\omega, j}
$$

that is, $Q_{\omega, \text { genuine }}$ is the interval where the mean $\boldsymbol{\mu}_{\omega}$ is located. In Figure 4 we give an illustration of a $b$-bit APQ.

The adaptive offset $\varphi_{\omega}^{*}$ in (2) is determined by the background PDF $p_{\bar{\omega}}(\mathbf{v})$ as well as the genuine user PDF $p_{\omega}(\mathbf{v})$ : given both PDFs and an arbitrary offset $\varphi$, the theoretical detection rate $\delta$ and the FAR $\alpha$ at zero Hamming

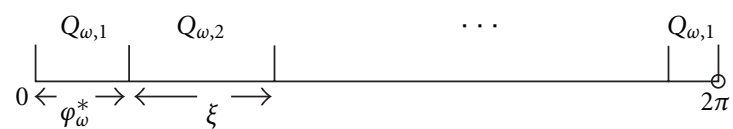

FIGURE 4: An illustration of a $b$-bit APQ in the phase domain, where $Q_{\omega, j}, j=1, \ldots, 2^{b}$ denotes the $j$ th quantization interval with width $\xi$, and offset angle $\varphi_{\omega}^{*}$. The first interval $Q_{\omega, 1}$ is wrapped.

distance threshold are

$$
\begin{aligned}
& \delta_{\omega}\left(Q_{\omega, \text { genuine }}\right)=\int_{Q_{\omega, \text { genuine }(b, \varphi)}} p_{\omega}(\mathbf{v}) d \mathbf{v}, \\
& \alpha_{\omega}\left(Q_{\omega, \text { genuine }}\right)=\int_{Q_{\omega, \text { genuine }}(b, \varphi)} p_{\bar{\omega}}(\mathbf{v}) d \mathbf{v} .
\end{aligned}
$$


Given that the background PDF is circularly symmetric, (5) is independent of $\varphi$. Thus, (5) becomes

$$
\alpha_{\omega}=2^{-b} \text {. }
$$

Therefore, the optimal $\varphi_{\omega}^{*}$ is determined by maximizing the detection rate in (4):

$$
\varphi_{\omega}^{*}=\arg \max _{\varphi} \delta_{\omega}
$$

After the $\varphi_{\omega}^{*}$ is determined, the quantization intervals are constructed from (2). Additionally, the detection rate of the $\mathrm{APQ}$ is

$$
\delta_{\omega}\left(Q_{\omega, \text { genuine }}\right)=\int_{Q_{\omega, \text { genuine }}\left(b, \varphi_{\omega}^{*}\right)} p_{\omega}(\mathbf{v}) d \mathbf{v} .
$$

Essentially, APQ has both equal-width and equalprobability intervals, with rotation offset $\varphi_{\omega}^{*}$ that maximizes the detection rate.

2.2. Simulations on Independent Gaussian Densities. We investigate the APQ performances on synthetic data, in a particular case where the feature pairs have independent Gaussian densities. That is, the background PDF of both features are normalized as zero mean and unit variance, that is, $p_{\bar{\omega}, 1}=p_{\bar{\omega}, 2}=N(v, 0,1)$. Similarly, the genuine user PDFs are $p_{\omega, 1}(v)=N\left(v, \mu_{\omega, 1}, \sigma_{\omega, 1}\right)$ and $p_{\omega, 2}(v)=N\left(v, \mu_{\omega, 2}, \sigma_{\omega, 2}\right)$. Since the two features are independent, the two-dimensional joint background PDF $p_{\bar{\omega}}(\mathbf{v})$ and the joint genuine user PDF $p_{\omega}(\mathbf{v})$ are

$$
\begin{aligned}
& p_{\bar{\omega}}(\mathbf{v})=p_{\bar{\omega}, 1} \cdot p_{\bar{\omega}, 2}, \\
& p_{\omega}(\mathbf{v})=p_{\omega, 1} \cdot p_{\omega, 2} .
\end{aligned}
$$

According to (6), the FAR for a $b$-bit APQ is fixed to $2^{-b}$. Therefore, we only have to investigate the detection rate in (8) regarding the genuine user PDF $p_{\omega}$, defined by the $\mu$ and $\sigma$ values. In Figure 5, we show the detection rate $\delta_{\omega}$ of the $b$-bit APQ $(b=1,2,3,4)$, when $p_{\omega}(\mathbf{v})$ is modeled as $\sigma_{\omega, 1}=\sigma_{\omega, 2}=0.2 ; \sigma_{\omega, 1}=\sigma_{\omega, 2}=0.8 ; \sigma_{\omega, 1}=0.8, \sigma_{\omega, 2}=0.2$, at various $\left\{\mu_{\omega, 1}, \mu_{\omega, 2}\right\}$ locations for optimal $\varphi_{\omega}^{*}$. The white pixels represent high values of the detection rate whilst the black pixels represent low values. The $\delta_{\omega}$ appears to depend more on how far the features are from the origin than on the direction of the features. This is due to the rotation adaptive property. In general, the $\delta_{\omega}$ is higher when the genuine user PDF has smaller $\sigma_{\omega}$ and larger $\mu_{\omega}$ for both features. Either decreasing the $\mu_{\omega}$ or increasing the $\sigma_{\omega}$ deteriorates the performance.

To generalize such property, we define a Mahalanobis distance $d_{\omega, i}$ for feature $i$ as

$$
d_{\omega, i}=\operatorname{abs}\left(\frac{\mu_{\omega, i}}{\sigma_{\omega, i}}\right) .
$$

Given the Mahalanobis distances $d_{\omega, 1}, d_{\omega, 2}$ of two features, we define $\bar{d}_{\omega}$ for this feature pair as

$$
\bar{d}_{\omega}=\sqrt{d_{\omega, 1}^{2}+d_{\omega, 2}^{2}}
$$

In Figure 6 we give some simulation results for the relation between $\bar{d}_{\omega}$ and $\delta_{\omega}$. The parameters $\mu$ and $\sigma$ for the genuine user PDF $p_{\omega}$ are modeled as four $\sigma$ combinations at various $\mu$ locations. For every $\mu-\sigma$ setting, we plot its $\bar{d}_{\omega}$ and $\delta_{\omega}$. We observe that the detection rate $\delta_{\omega}$ tends to increase when the feature pair Mahalanobis distance $\bar{d}_{\omega}$ increases, although not always monotonically.

We further compare the detection rate of APQ to that of the one-dimensional fixed quantizer (FQ) [17]. In order to compare with the 2-bit APQ at the same FAR, we choose a 1 -bit FQ $(b=1)$ for every feature dimension. In Figure 7 we show the ratio of their detection rates $\left(\delta_{\mathrm{APQ}} / \delta_{\mathrm{FQ}}\right)$ at various $\mu-\sigma$ values. The white pixels represent high values whilst the black pixels represent low values. It is observed that APQ consistently outperforms FQ, especially when the mean of the genuine user PDF is located far away from the origin and close to the FQ boundary, namely, the $v_{1}$-axis and $v_{2}$-axis. In fact, the two 1-bit FQ works as a special case of the 2-bit $\mathrm{APQ}$, with $\varphi_{\omega}^{*}=0$.

\section{Biometric Binary String Extraction}

The APQ can be directly applied to two-dimensional features, such as Iris [22], while for arbitrary features, we have the freedom to pair the features. In this section, we first formulate the pairing problem, which in practice is difficult to solve. Therefore, we simplify this problem and then propose a long-short pairing strategy (LS) with low computational complexity.

3.1. Problem Formulation. The aim for extracting biometric binary string is for a genuine user $\omega$ who has $D$ features, we need to determine a strategy to pair these $D$ features into $D / 2$ pairs, in such way that the entire $L$-bit binary string $(L=$ $b \times D / 2$ ) obtains optimal classification performance, when every feature pair is quantized by a $b$-bit APQ. Assuming that the $D / 2$ feature pairs are statistically independent, we know from [19] that when applying a Hamming distance classifier, zero Hamming distance threshold gives a lower bound for both the detection rate and the FAR. Therefore, we decide to optimize this lower bound classification performance.

Let $c_{\omega, k},(k=1, \ldots, D / 2)$ be the $k$ th pair of feature indices, and $\left\{c_{\omega, k}\right\}$ a valid pairing configuration containing $D / 2$ feature index pairs such that every feature index only appears once. For instance, $c_{\omega, k}=(1,1)$ is not valid because it contains the same feature and therefore cannot be included in $\left\{c_{\omega, k}\right\}$. Also, $\left\{c_{\omega, k}\right\}=\{(1,2),(1,3)\}$ is not a valid pairing configuration because the index value " 1 " appears twice. The overall FAR $\left(\alpha_{\omega}\right)$ and the overall detection rate $\left(\delta_{\omega}\right)$, at zero Hamming distance threshold are

$$
\begin{aligned}
& \alpha_{\omega}\left(\left\{c_{\omega, k}\right\}\right)=\prod_{k=1}^{D / 2} \alpha_{\omega, k}\left(c_{\omega, k}\right), \\
& \delta_{\omega}\left(\left\{c_{\omega, k}\right\}\right)=\prod_{k=1}^{D / 2} \delta_{\omega, k}\left(c_{\omega, k}\right),
\end{aligned}
$$



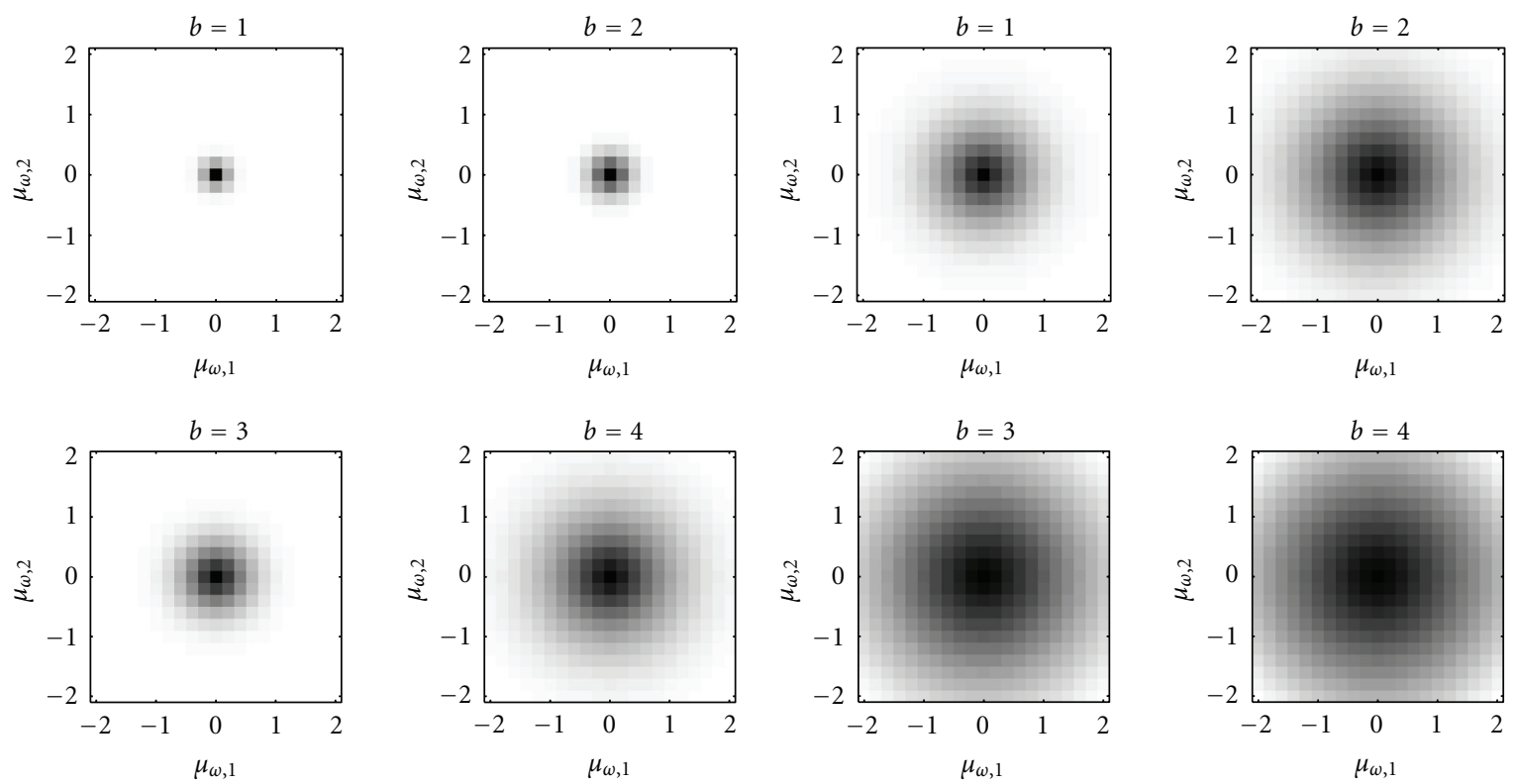

(a)

(b)
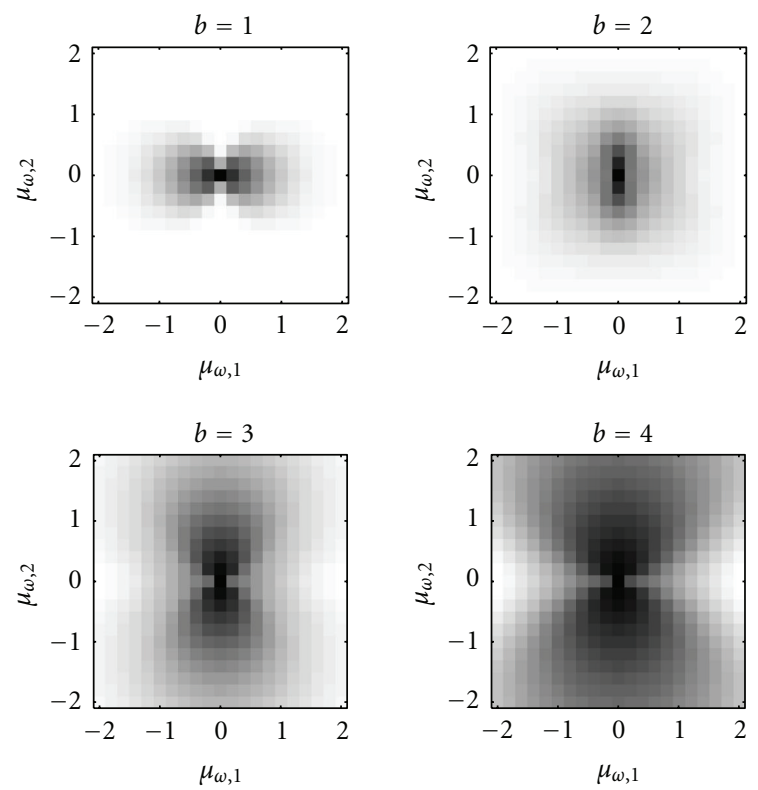

(c)

Figure 5: The detection rate of the $b$-bit APQ $(b=1,2,3,4)$, when $p_{\omega}(\mathbf{v})$ is modeled as (a) $\sigma_{\omega, 1}=\sigma_{\omega, 2}=0.2 ;(\mathrm{b}) \sigma_{\omega, 1}=\sigma_{\omega, 2}=0.8$; (c) $\sigma_{\omega, 1}=0.8, \sigma_{\omega, 2}=0.2$, at various $\left\{\mu_{\omega, 1}, \mu_{\omega, 2}\right\}$ locations: $\mu_{\omega, 1}, \mu_{\omega, 2} \in[-22]$. The detection rate ranges from 0 (black) to 1 (white).

where $\alpha_{\omega, k}$ and $\delta_{\omega, k}$ are the FAR and the detection rate for the $k$ th feature pair, computed from (6) and (8). Furthermore, according to (6), $\alpha_{\omega}$ becomes

$$
\alpha_{\omega}=2^{-L}
$$

which is independent of $\left\{c_{\omega, k}\right\}$. Therefore, we only need to search for a user-specific pairing configuration $\left\{c_{\omega, k}^{*}\right\}$, that maximizes the overall detection rate in (13). Solving the optimization problem is formulated as

$$
\left\{c_{\omega, k}^{*}\right\}=\arg \max _{\left\{c_{\omega, k}\right\}} \prod_{k=1}^{D / 2} \delta_{\omega}\left(c_{\omega, k}\right) .
$$

The detection rate $\delta_{\omega}$ given a feature pair $c_{\omega, k}$ is computed from (8). Considering that the performance at zero Hamming distance threshold indeed pinpoints the minimum FAR 


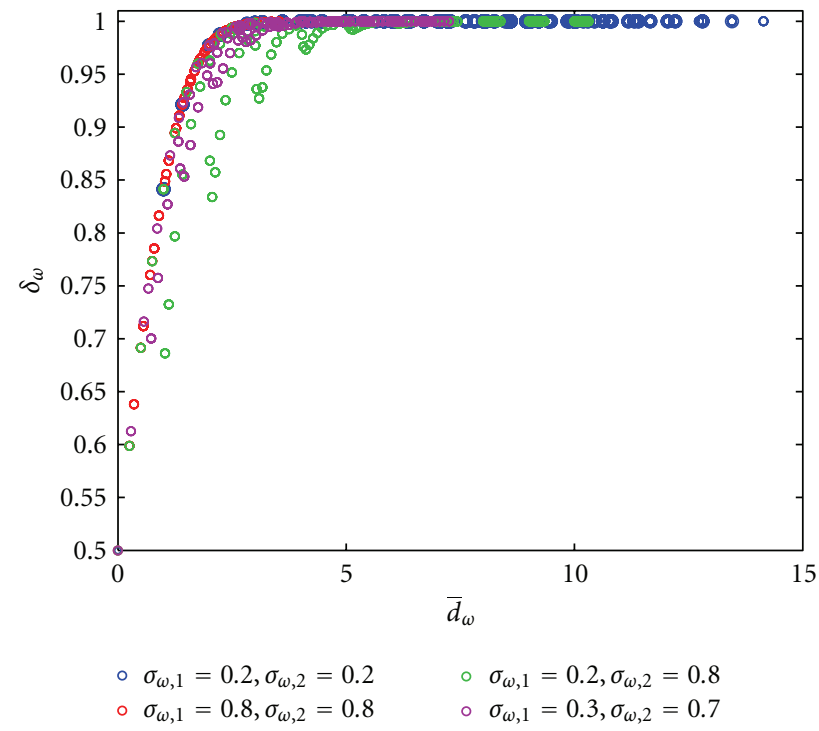

(a)

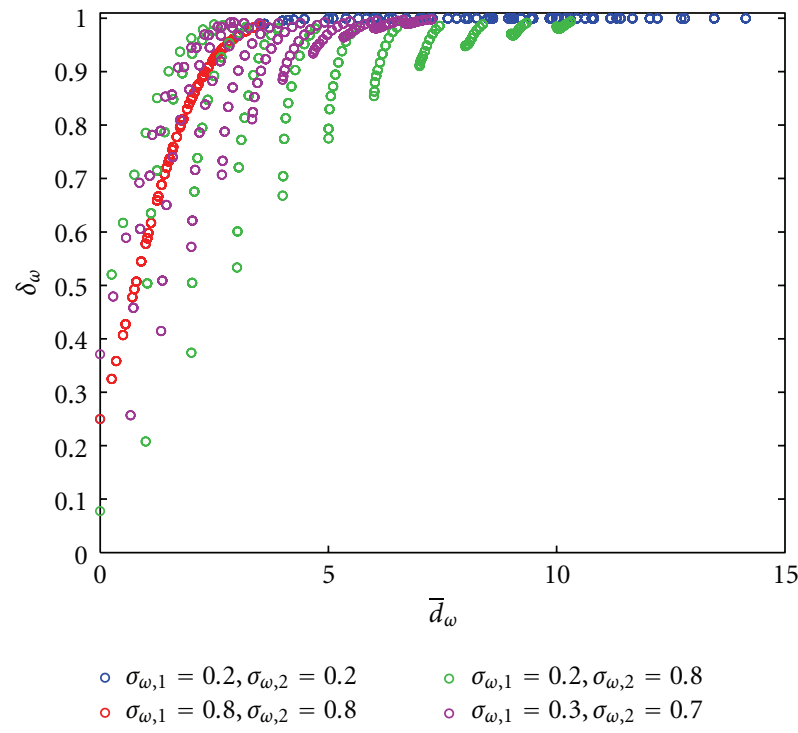

(b)

Figure 6: The relations between $\bar{d}_{\omega}$ and $\delta_{\omega}$ when the genuine user PDF $p_{\omega}$ is modeled as with $\mu_{\omega, 1}, \mu_{\omega, 2} \in[-22]$ and four $\sigma_{\omega, 1}, \sigma_{\omega, 2}$ settings. The result is shown as (a) 1-bit APQ; (b) 2-bit APQ.

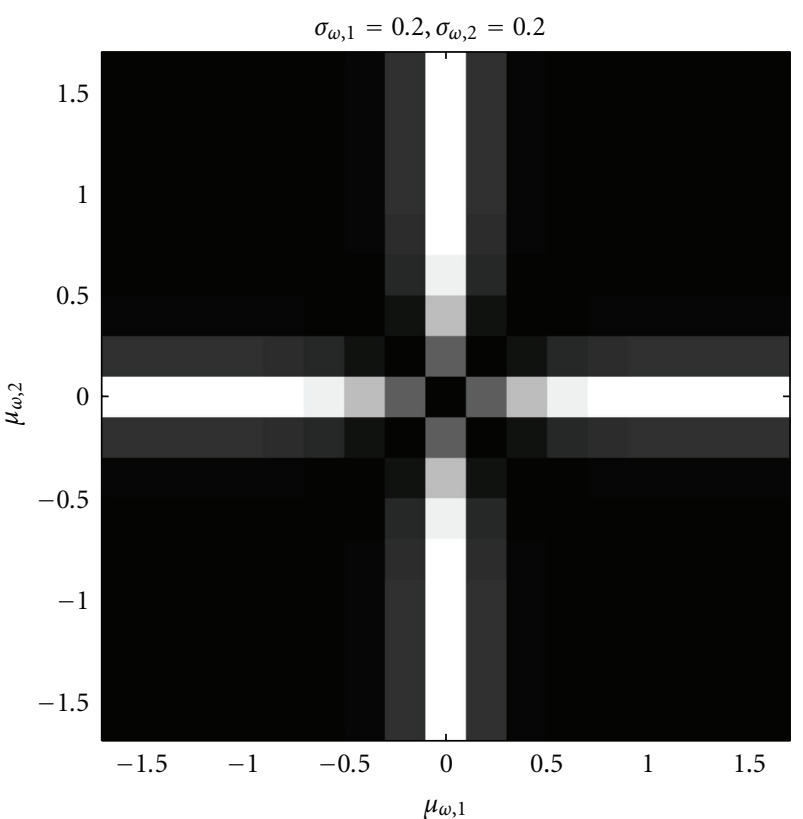

(a)

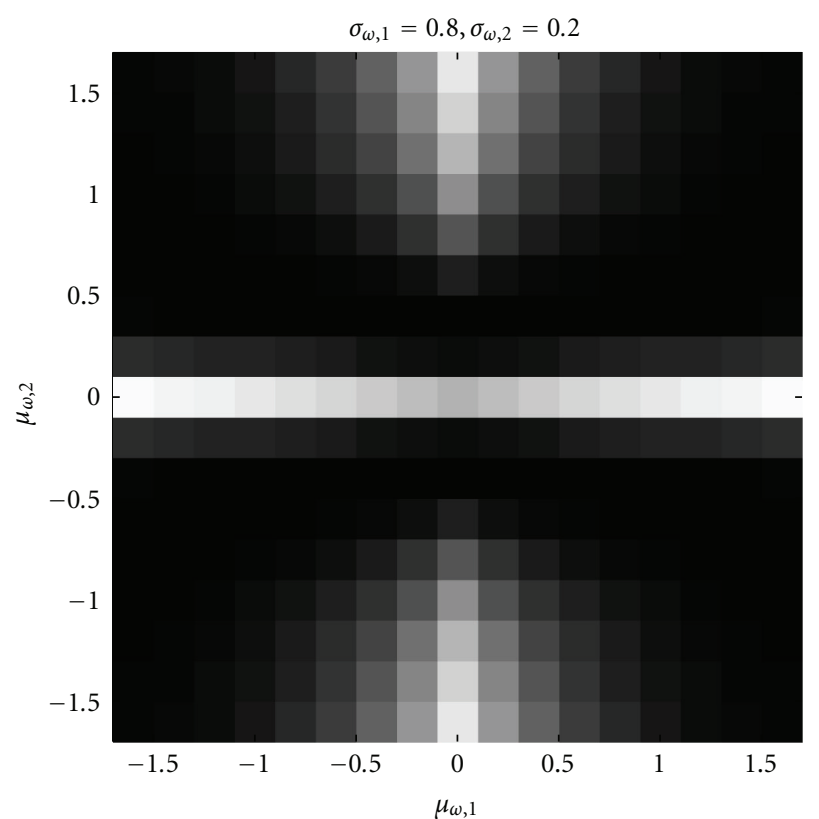

(b)

FIGURE 7: The detection rate ratio $\delta_{\mathrm{APQ}} / \delta_{\mathrm{FQ}}$ of the 2-bit $\mathrm{APQ}$ to the 1-bit FQ $(b=1)$, when $p_{\omega}(\mathbf{v})$ is modeled as $(\mathrm{a}) \sigma_{\omega, 1}=\sigma_{\omega, 2}=0.2$; (b) $\sigma_{\omega, 1}=0.8, \sigma_{\omega, 2}=0.2$, with various $\mu_{\omega, 1}, \mu_{\omega, 2}$ locations: $\mu_{\omega, 1}, \mu_{\omega, 2} \in[-1.61 .6]$. The detection rate ratio ranges from 1 (black) to 2 (white).

and detection rate value on the receiver operating characteristic curve (ROC), optimizing such point in (15) essentially provides a maximum lower bound for the ROC curve.

3.2. Long-Short Pairing. There are two problems in solving (15): first, it is often not possible to compute $\delta_{c_{\omega, k}}$ in (8), due to the difficulties in estimating the genuine user PDF $p_{\omega}$. Additionally, even if the $\delta_{\omega_{\omega, k}}$ can be accurately estimated, a brute-force search would involve $2^{-D / 2} D ! /(D / 2)$ ! evaluations of the overall detection rate, which renders a brute-force search unfeasible for realistic values of $D$. Therefore, we propose to simplify the problem definition in (15) as well as the optimization searching approach. 


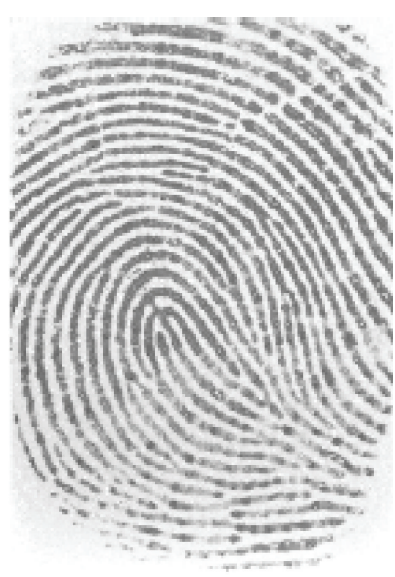

(a)

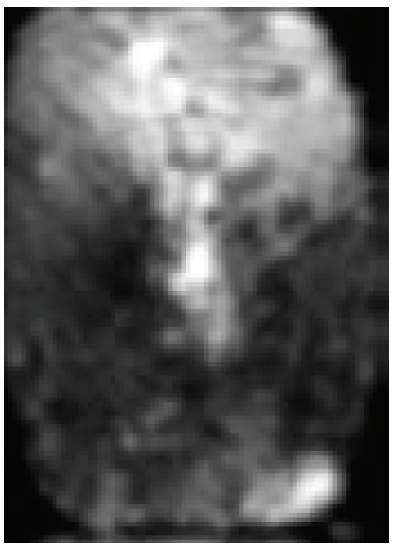

(c) 0

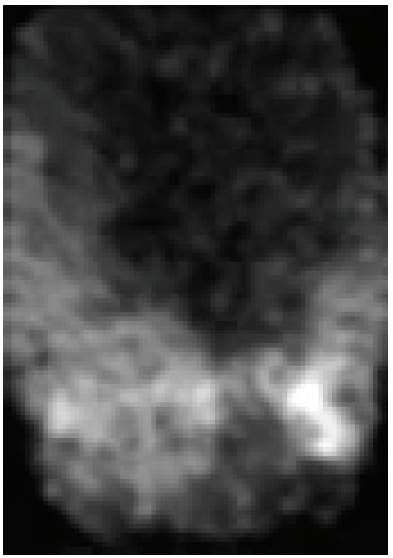

(e) $\frac{1}{2} \pi$

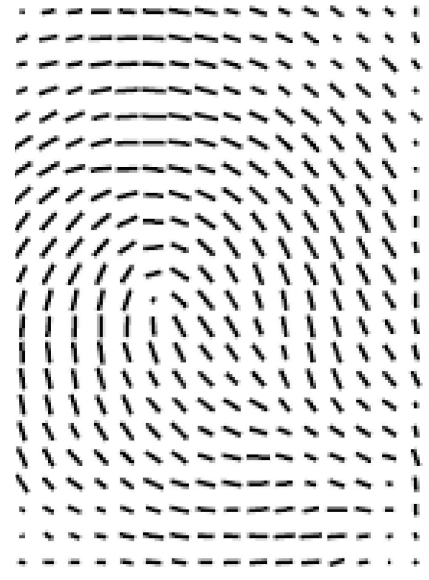

(b)

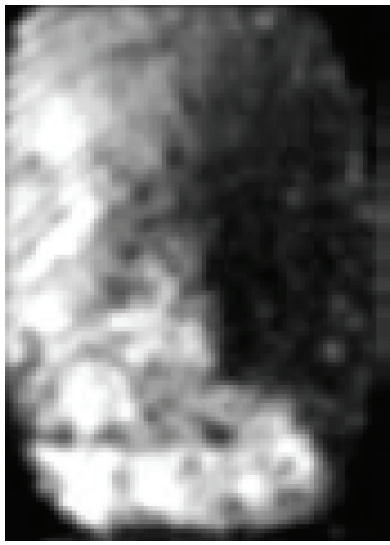

(d) $\frac{1}{4} \pi$

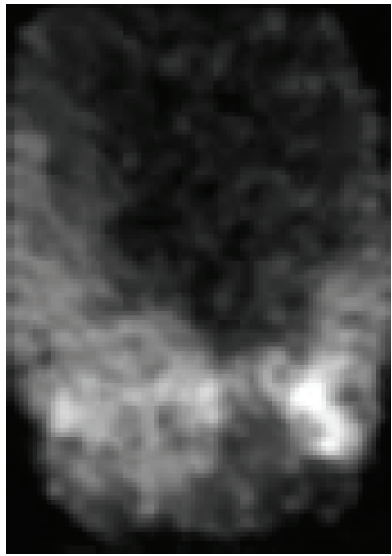

(f) $\frac{3}{4} \pi$

Figure 8: (a) Fingerprint image, (b) directional field, and (c)-(f) the absolute values of Gabor responses for different orientations $\theta$.

Simplified Problem Definition. In Section 2.2 we observed a useful relation between $\bar{d}$ and $\delta$ for the APQ: A feature pair with a higher $\bar{d}$ would approximately also obtain a higher detection rate $\delta_{\omega}$ for APQ. Therefore, we simplify (15) into

$$
\left\{c_{\omega, k}^{*}\right\}=\arg \max _{\left\{c_{\omega, k}\right\}} \prod_{k=1}^{D / 2} \bar{d}_{\omega}\left(c_{\omega, k}\right),
$$

with $\bar{d}_{\omega}\left(c_{\omega, k}\right)$ defined in (11). Furthermore, instead of brute force searching, we propose a simplified optimization searching approach: the long-short (LS) pairing strategy.

Long-Short (LS) Pairing. For the genuine user $\omega$, sort the set $\left\{d_{\omega, i}=\operatorname{abs}\left(\mu_{\omega, i} / \sigma_{\omega, i}\right): i=1, \ldots, D\right\}$ from largest to smallest into a sequence of ordered feature indices $\left\{I_{\omega, 1}, I_{\omega, 2}, \ldots, I_{\omega, D}\right\}$. 


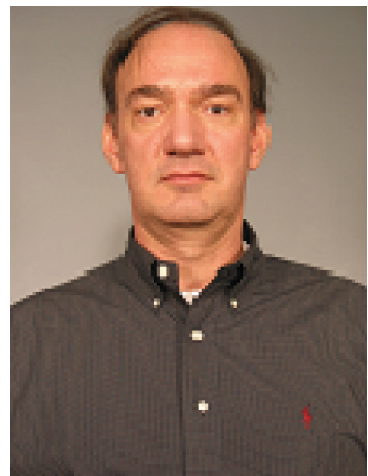

(a)

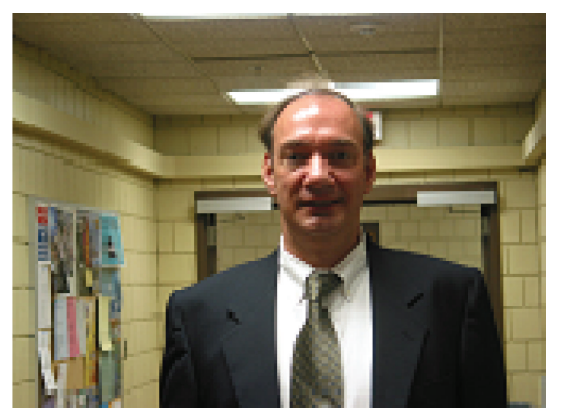

(b)

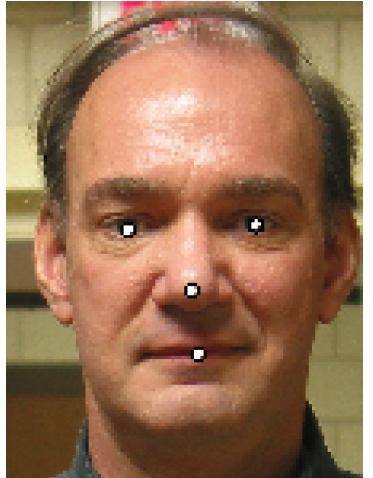

(c)

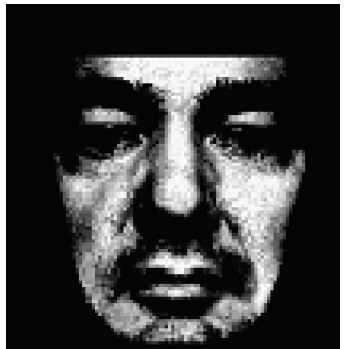

(d)

Figure 9: (a) Controlled image, (b) uncontrolled image, (c) landmarks, and (d) the region of interest (ROI).

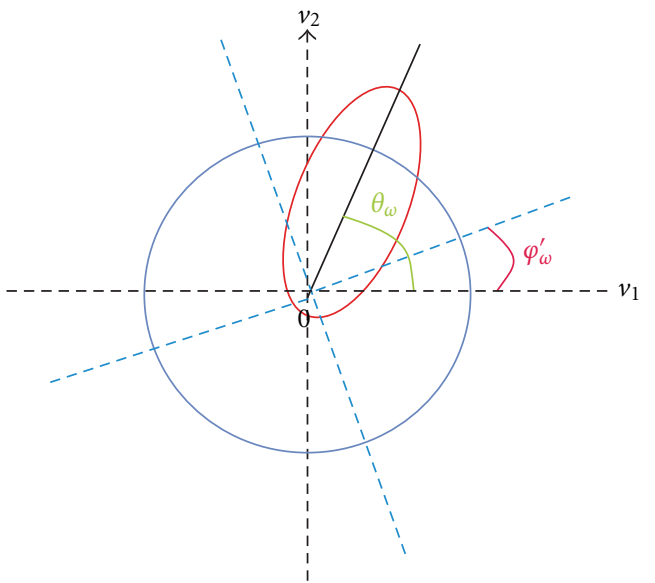

Figure 10: An example of a 2-bit simplified APQ, with the background PDF (blue) and the genuine user PDF (red). The dashed lines are the quantization boundaries.

The index for the $k$ th feature pair is then

$$
c_{\omega, k}=\left(I_{\omega, k}, I_{\omega, D+1-k}\right), \quad k=1, \ldots, D / 2 .
$$

The computational complexity of the LS pairing is only $O(D)$. Additionally, it is applicable to arbitrary feature types and independent of the number of quantization bits $b$. Note that this LS pairing is similar to the pairing strategy proposed in [21], where Euclidean distances are used. In fact, there are other alternative pairing strategies, for instance greedy or long-long pairing [21]. However, in terms of the entire binary string performance, these methods are not as good as the approach presented in this paper, especially when $D$ is large. Therefore, in this paper, we choose the longshort pairing strategy, providing a compromise between the classification performance and computational complexity.

\section{Experiments}

In this section we test the pairwise phase quantization (LS + $\mathrm{APQ}$ ) on real data. First we present a simplified APQ, which

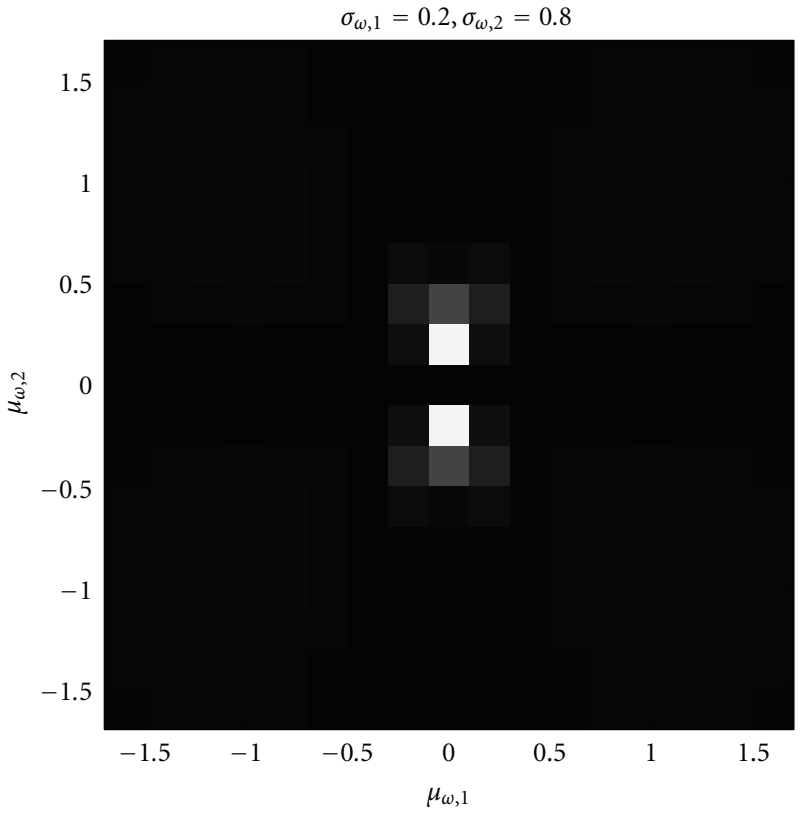

FIGURE 11: The detection rate ratio between the original 2-bit APQ and the simplified APQ, when $p_{\omega}(\mathbf{v})$ is modeled as $\sigma_{\omega, 1}=0.2, \sigma_{\omega, 2}=$ 0.8 , with various $\mu_{\omega, 1}, \mu_{\omega, 2}$ locations: $\mu_{\omega, 1}, \mu_{\omega, 2} \in[-1.6$ 1.6]. The detection rate ratio scale is $\left[\begin{array}{ll}1 & 2.2\end{array}\right]$.

is employed in all the experiments. Afterwards, we verify the relation between $\bar{d}$ and $\delta$ for real data. We also show some examples of LS pairing results. Then we investigate the verification performances while varying the input feature dimensions $(D)$ and the number of quantization bits per feature pair $(b)$. The results are further compared to the onedimensional fixed quantization (1D FQ) [17] as well as the the FQ in combined with the DROBA bit allocation principle $(\mathrm{FQ}+\mathrm{DROBA})$.

4.1. Experimental Setup. We tested the pairwise phase quantization on two real data sets: the FVC2000(DB2) fingerprint database [23] and the FRGC(version 1) face database [24]. 


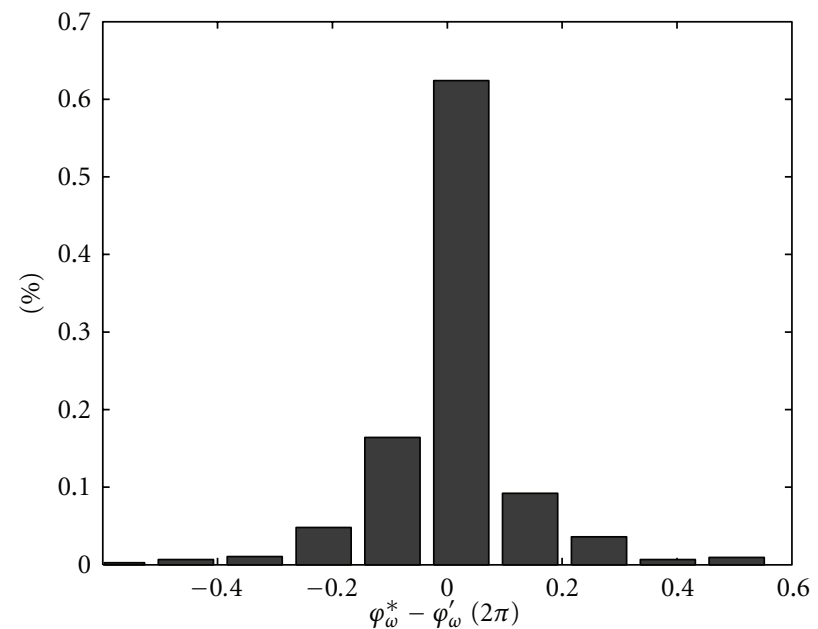

(a)

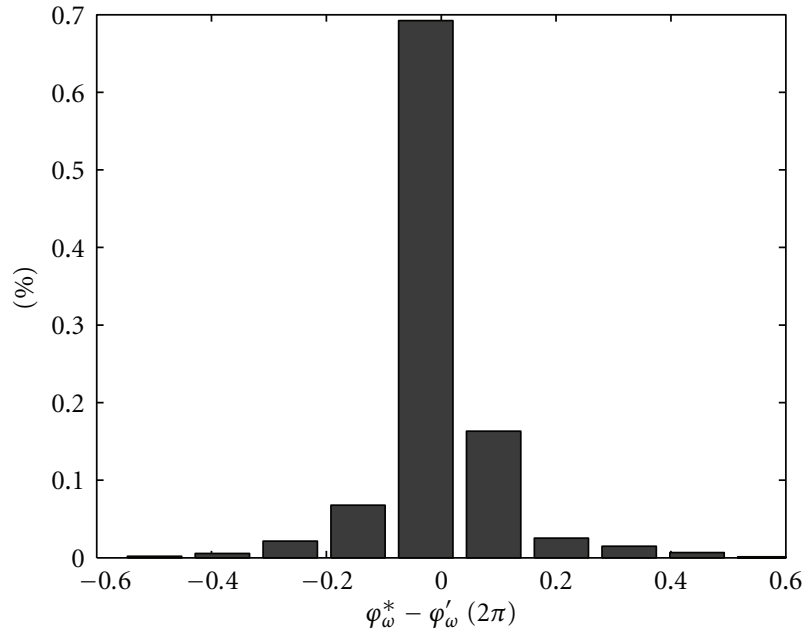

(b)

Figure 12: The differences of the rotation angle between the original APQ and the simplified APQ $\left(\varphi_{\omega}^{*}-\varphi_{\omega}^{\prime}\right)$, computed from 50 feature pairs, for (a) FVC2000 and (b) FRGC.

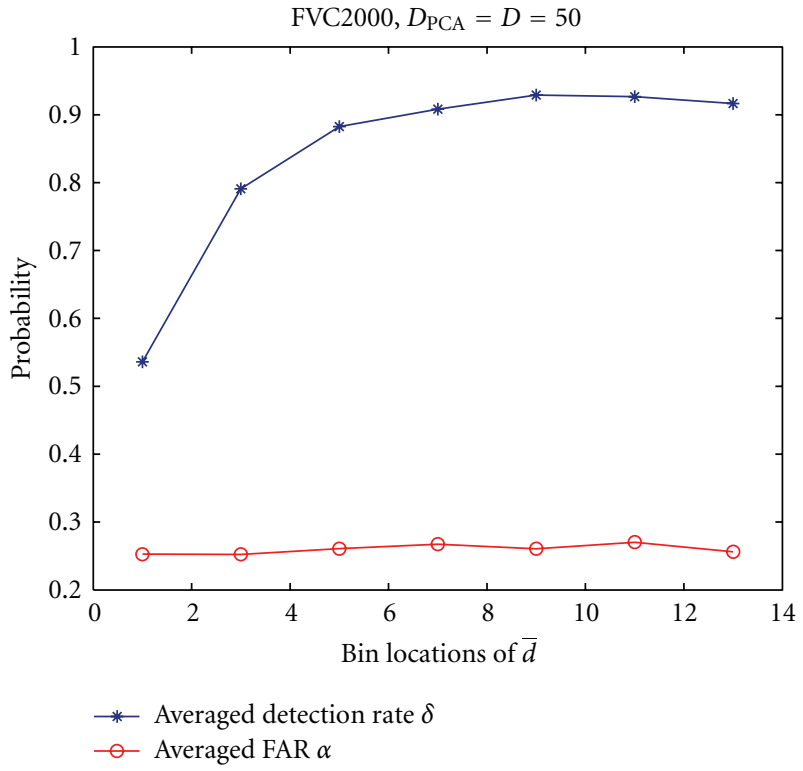

(a)

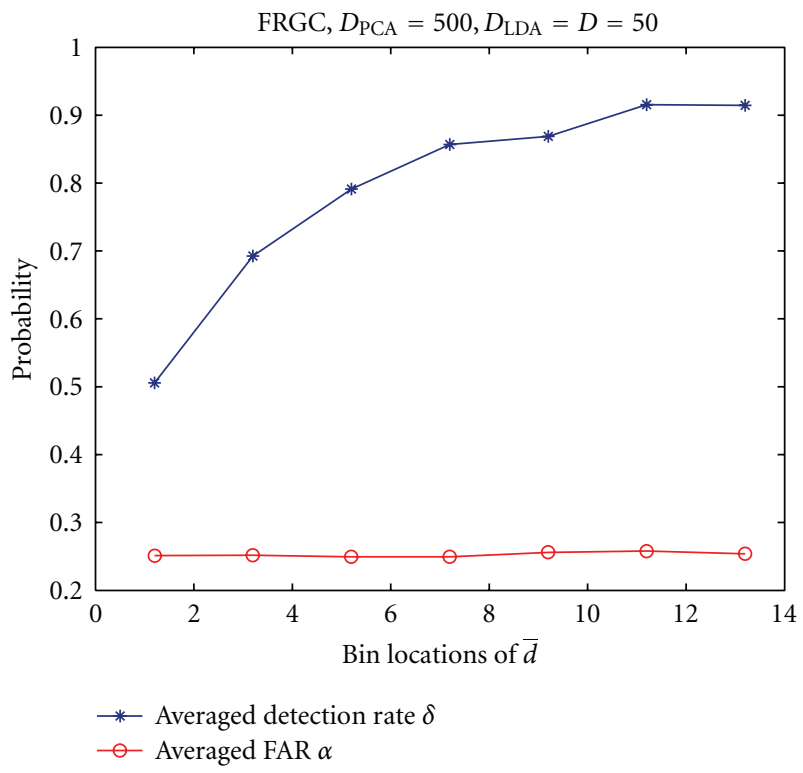

(b)

FIGURE 13: The averaged value of the detection rate and the FAR that correspond to the bins of $\bar{d}$, derived from the random pairing and the 2-bit APQ, for (a) FVC2000 and (b) FRGC.

(i) FVC2000: The FVC2000(DB2) fingerprint data set contains 8 images of 110 users. The features were extracted in a fingerprint recognition system that was used in [10]. As illustrated in Figure 8, the raw features contain two types of information: the squared directional field in both $x$ and $y$ directions and the Gabor response in 4 orientations $(0, \pi / 4, \pi / 2,3 \pi / 4)$. Determined by a regular grid of 16 by 16 points with spacing of 8 pixels, measurements are taken at 256 positions, leading to a total of 1536 elements. (ii) FRGC: The FRGC(version 1) face data set contains 275 users with a different number of images per user, taken under both controlled and uncontrolled conditions. The number of samples $s$ per user ranges from 4 to 36 . The image size was $128 \times 128$. From that a region of interest (ROI) with 8762 pixels was taken as illustrated in Figure 9.

A limitation of biometric compression or protection is that it is not possible to conduct the user-specific image 

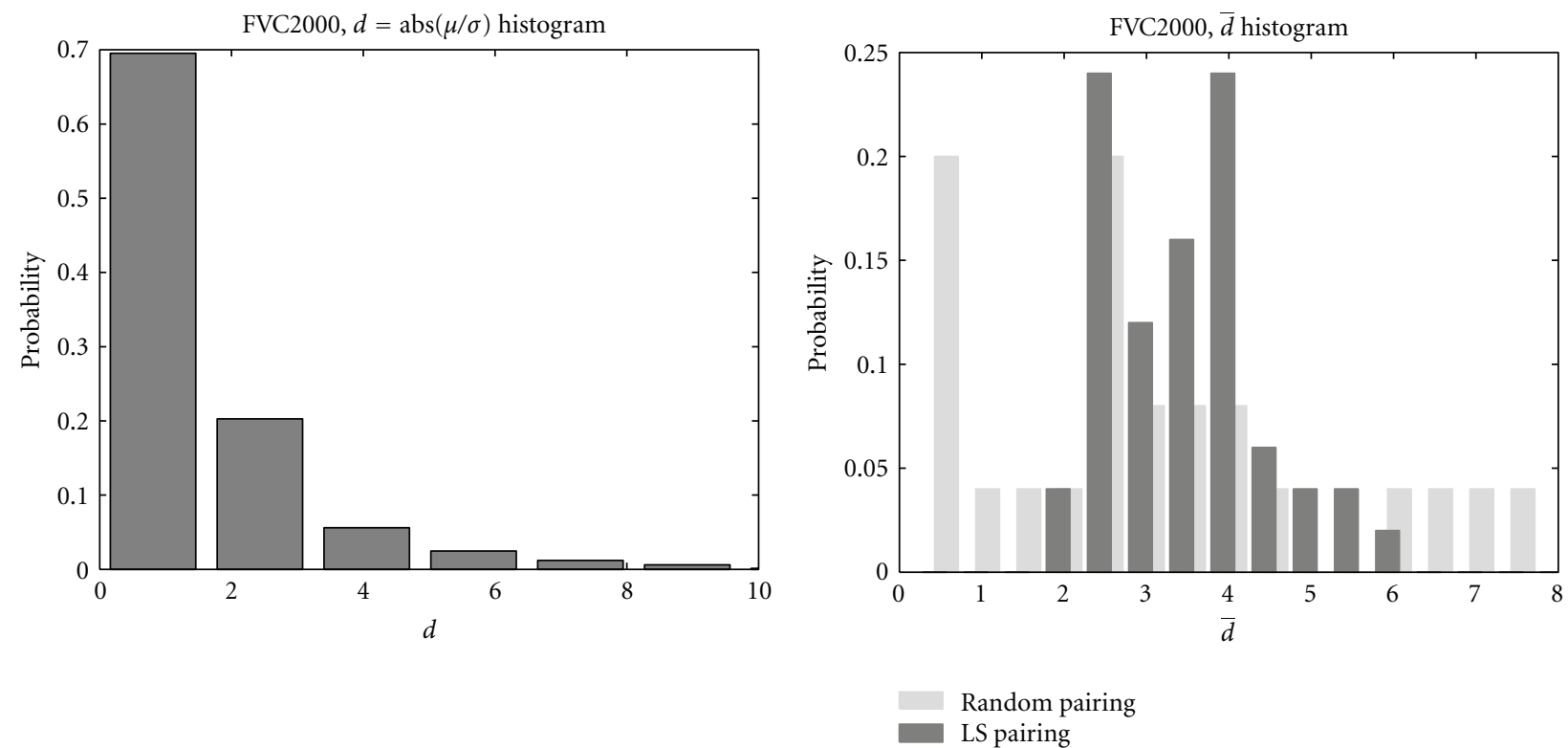

(a)

(b)

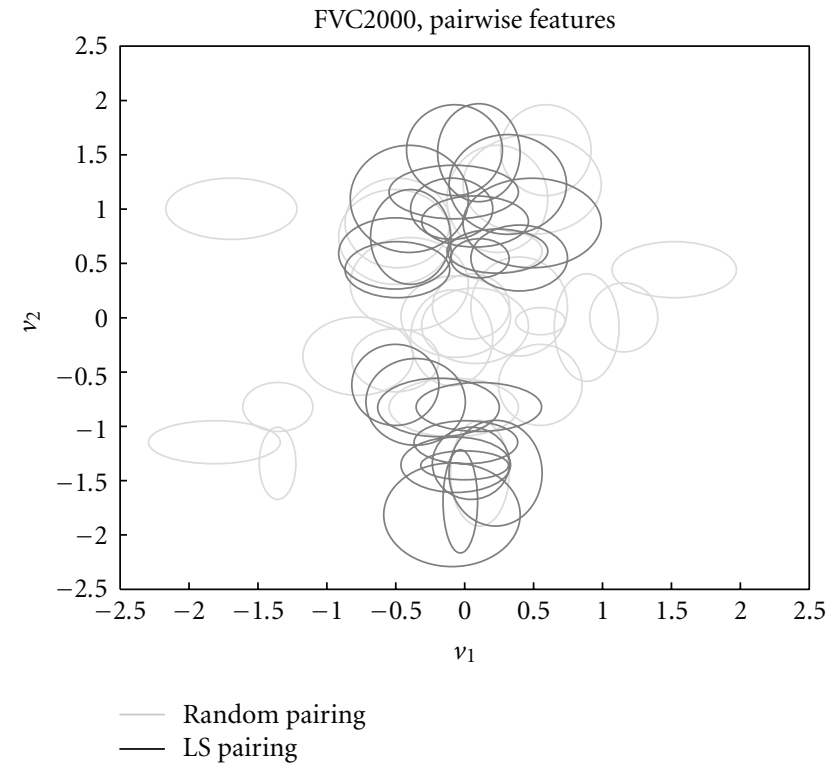

(c)

Figure 14: An example of the LS pairing performance on FVC2000, at $D=50$. (a) the histogram of $d=\operatorname{abs}(\mu / \sigma)$; (b) the histogram of $\bar{d}$ for pairwise features and (c) an illustration of the pairwise features as independent Gaussian density, from both LS and random pairing.

alignment, because the image or other alignment information cannot be stored. Therefore, in this paper, we applied basic absolute alignment methods: the fingerprint images are aligned according to a standard core point position; the face images are aligned according to a set of four standard landmarks, that is, eyes, nose and mouth.

We randomly selected different users for training and testing and repeated our experiments with a number of trials. The data division is described in Table 2, where $s$ is the number of samples per user that varies in the experiments.

Our experiments involved three steps: training, enrollment, and verification. (1) In the training step, we first
TABLE 2: Data division: number of users $\times$ number of samples per user(s), and the number of trials for FVC2000 and FRGC. The $s$ is a parameter that varies in the experiments.

\begin{tabular}{lcccc}
\hline & Training & Enrollment & Verification & Trials \\
\hline FVC2000 & $80 \times 8$ & $30 \times 6$ & $30 \times 2$ & 20 \\
FRGC & $210 \times s$ & $65 \times 2 s / 3$ & $65 \times s / 3$ & 5 \\
\hline
\end{tabular}

applied a combined PCA/LDA method [25] on a training set. The obtained transformation was then applied to both the enrollment and verification sets. We assume that the 


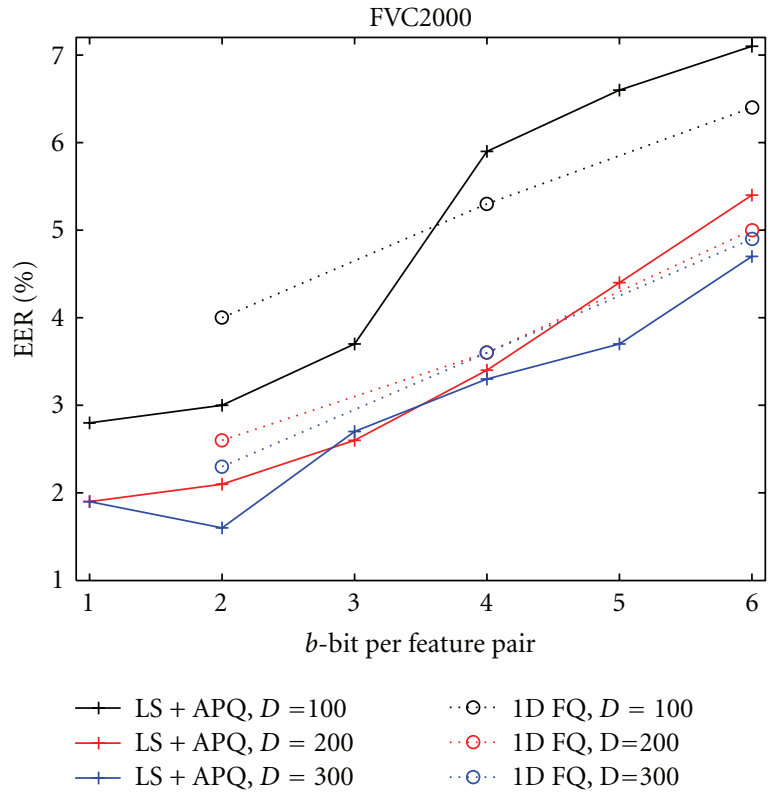

(a)

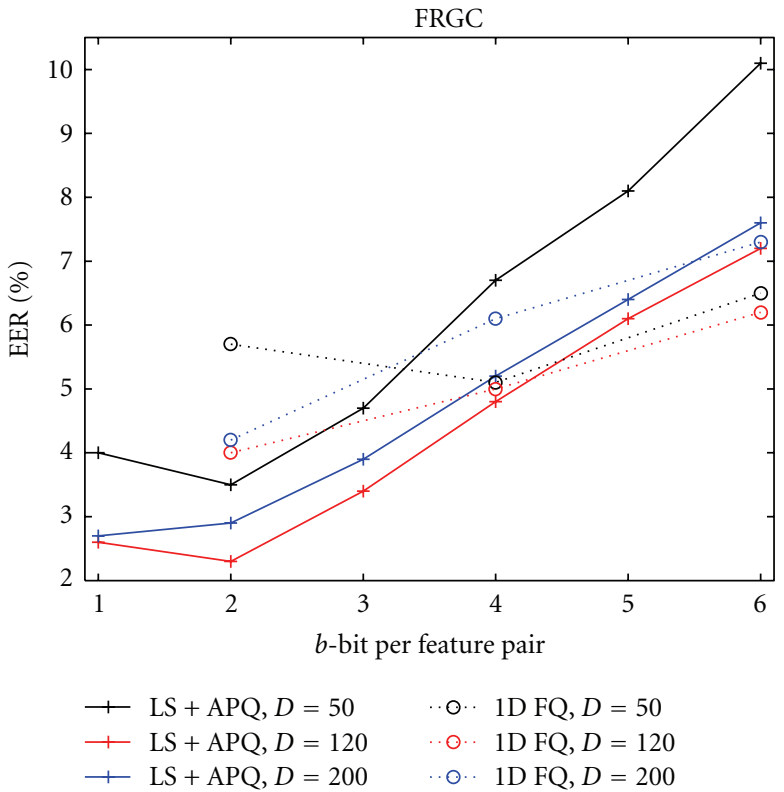

(b)

FIGURE 15: The EER performances of $b$-bit $\left(b \in\left[\begin{array}{ll}1 & 6\end{array}\right]\right)$ LS + APQ at various feature dimensionality $D$, as compared with the $b / 2$-bit $1 D$ FQ ( $b$-bit per feature pair), for (a) FVC2000, and (b) FRGC.

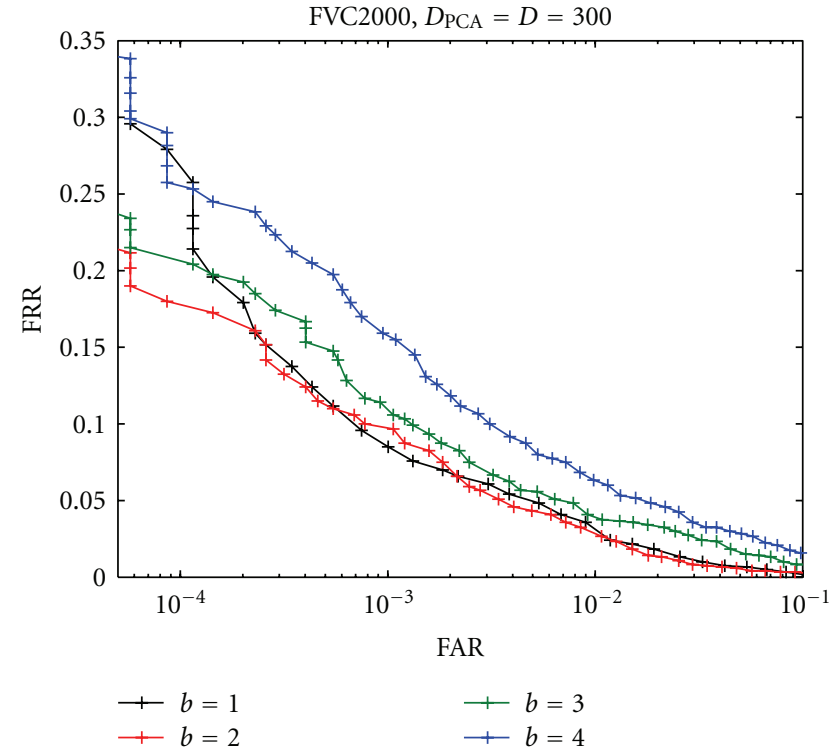

(a)

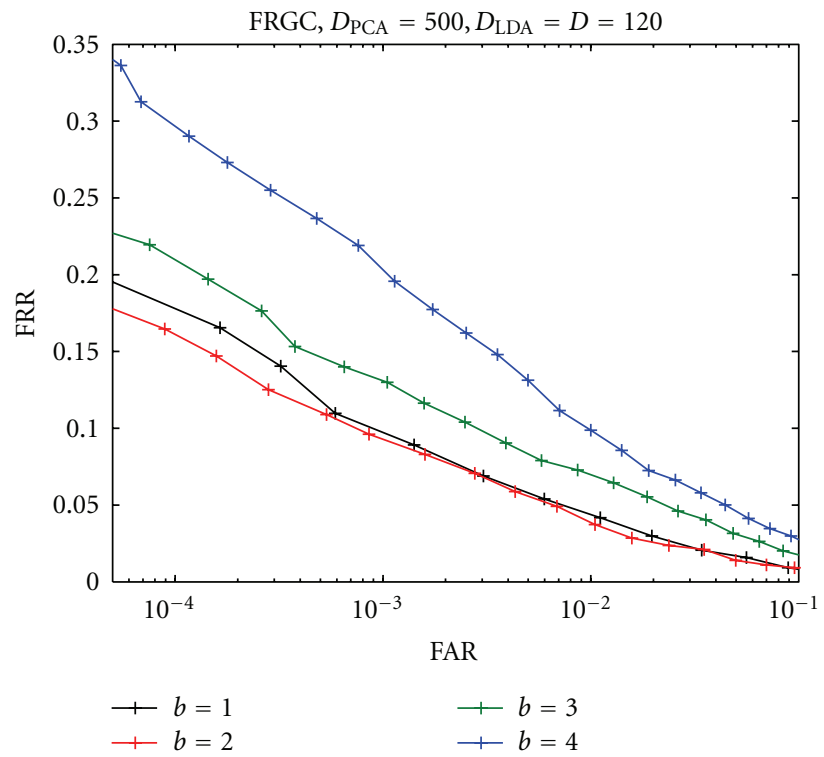

(b)

FIgURE 16: An example of the FAR/FRR performances (FAR in logarithm) of LS + APQ, with $b$ from 1 to 4, for (a) FVC2000 and (b) FRGC.

measurements have a Gaussian density, thus after the PCA transformation, the extracted features are assumed to be statistically independent. The goal of applying PCA/LDA in the training step is to extract independent features so that by pairing them we could subsequently obtain independent feature pairs, which meet our problem requirements. Note that for FVC2000, since we have only 80 users in the training set, applying LDA results in very limited number of features (e.g., $D \leq 79$ ). Therefore, we relax the independency requirement for the genuine user by applying only the PCA transformation. (2) In the enrollment step, for every genuine user $\omega$, the LS pairing was first applied, resulting in the user-specific pairing configuration $\left\{c_{\omega, k}^{*}\right\}$. The pairwise features were further quantized through a $b$-bit APQ with the adaptive angle $\left\{\varphi_{\omega, k}^{*}\right\}$, and assigned with a Gray code [26]. The concatenation of the codes from $D / 2$ feature pairs 


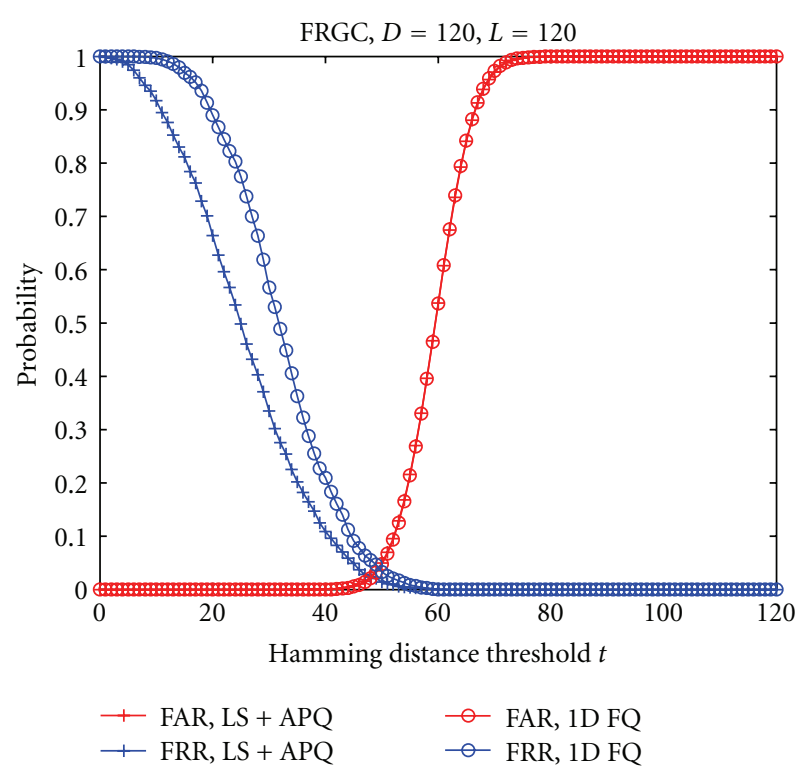

FIGURE 17: An example of the FAR/FRR performances of LS + APQ and $1 \mathrm{D} \mathrm{FQ}$, at $D=120, L=120$ for FRGC.

formed the $L$-bit target binary string $S_{\omega}$. Both $S_{\omega}$ and the quantization information $\left(\left\{c_{\omega, k}^{*}\right\},\left\{\varphi_{\omega, k}^{*}\right\}\right)$ were stored for each genuine user. (3) In the verification step, the features of the query user were quantized and coded according to the quantization information $\left(\left\{c_{\omega, k}^{*}\right\},\left\{\varphi_{\omega, k}^{*}\right\}\right)$ of the claimed identity, leading to a query binary string $S^{\prime}$. Finally, the decision was made by comparing the Hamming distance between the query and the target string.

4.2. Simplified $A P Q$. In practice, computing the optimal offset angle $\varphi_{\omega}^{*}$ for APQ in (7) is difficult, because it is hard to find a closed-form solution $\varphi_{\omega}^{*}$. Besides, it is often impossible to accurately estimate the underlying genuine user PDF $p_{\omega}$, due to the limited number of available samples per user. Therefore, instead of $\varphi_{\omega}^{*}$, we propose an approximate solution $\varphi_{\omega}^{\prime}$. For genuine user $\omega$, let the mean of the twodimensional feature vector be $\left\{\mu_{\omega, 1}, \mu_{\omega, 2}\right\}$, and its phase be $\bar{\theta}_{\omega}=\operatorname{angle}\left(\mu_{\omega, 1}, \mu_{\omega, 2}\right)$, the approximate offset angle $\varphi_{\omega}^{\prime}$ is then computed as

$$
\varphi_{\omega}^{\prime}=\bar{\theta}_{\omega}-\frac{\xi}{2}
$$

where $\xi=2 \pi / 2^{b}$. We give an illustration of computing $\varphi_{\omega}^{\prime}$ in Figure 10. The approximate solution $\varphi_{\omega}^{\prime}$ in fact maximizes the product of two Euclidean distances, namely, the distance of the mean vector $\left\{\mu_{\omega, 1}, \mu_{\omega, 2}\right\}$ to both the lower and the higher genuine interval boundaries.

Note that when the two features have independent Gaussian density with equal standard deviation, $\varphi_{\omega}^{*}=\varphi_{\omega}^{\prime}$. Thus, in that case, the simplified APQ equals the original APQ. In Figure 11, we illustrates an example of the detection rate ratio between the simplified and the original $A P Q$, where both features are modeled as Gaussian with different standard deviations, for example, $\sigma_{\omega, 1}=0.2, \sigma_{\omega, 2}=0.8$.
The white pixels represent high values whilst the black pixels represent low values. Results show that the simplified APQ is only slightly worse than the original APQ when the mean of the two-dimensional feature $\left\{\mu_{\omega, 1}, \mu_{\omega, 2}\right\}$ is close to the origin. However, if we apply APQ after the LS pairing, we would expect that the overall selected pairwise features are located farther away from the origin. In such cases, the simplified APQ works almost the same as the original APQ. In Figure 12 we illustrate the differences of the rotation angle between the original APQ and the simplified APQ, computed from (7) and (18), respectively. These differences are computed from 50 feature pairs for both FVC2000 and FRGC. The results show that there is no much differences between the rotation angle. Additionally, the simplified APQ is much simpler, avoiding the problem of estimating the underlying genuine user PDF $p_{\omega}$. For these reasons, we employed this simplified APQ in all the following experiments (Section 4.3 to Section 4.5).

4.3. $A P Q \bar{d}-\delta$ Property. In this section we test the relation between the APQ detection rate $\delta_{\omega}$ and the pairwise feature's distance $\bar{d}_{\omega}$ on both data sets. The goal is to see whether the real data exhibit the same $\bar{d}_{\omega}-\delta_{\omega}$ property as we found with synthetic data in Section 2.2: the feature pairs with higher $\overline{d_{\omega}}$ obtains higher detection rate $\delta_{\omega}$.

During the enrollment, for every genuine user, we conducted a random pairing. For every feature pair, we computed their $\bar{d}_{\omega}$ value according to (11). Afterwards, we applied the $b$-bit APQ quantizer to every feature pair. In the verification, for every feature pair, we computed the Hamming distance between the $b$-bits from the genuine user and the $b$-bits from the imposters; that is, we count as a detection if the $b$-bit genuine query string obtains zero Hamming distance as compared to the target string. Similarly, we count as a false acceptance if the $b$-bit imposter query string obtains zero Hamming distance as compared to the target string. We then repeated this process over all feature pairs as well as all genuine users, in order to ensure that the results we obtain are neither user or feature biased. Finally, in Figure 13, we plot the relations between the $\bar{d}_{\omega}$ and the $\delta_{\omega}$. The points we plot are averaged according to the bins of $\bar{d}_{\omega}$, when $b=2$. Results show that for the real data, the larger $\bar{d}_{\omega}$ is, consistently the higher detection rate we obtain. Additionally, the FAR performance is indeed independent of pairing and equals the theoretical value $2^{-b}$.

4.4. LS Pairing Performance. In this section we test the LS pairing performances. We give an example of FVC2000 at $D=50$. Figure 14(a) shows the histogram of $d$ for all single features over all the genuine users. Around $70 \%$ of them are close to zero, suggesting low quality features. After LS pairing, the histogram of the pairwise $\bar{d}$ values are shown in Figure 14(b), as compared with the random pairing. In Figure 14(c), we illustrate the 25 pairwise features in terms of independent Gaussian densities, for one specific genuine user. Figures 14(b) and 14(c) shows that after LS pairing, a large proportion of feature pairs have relatively moderate 


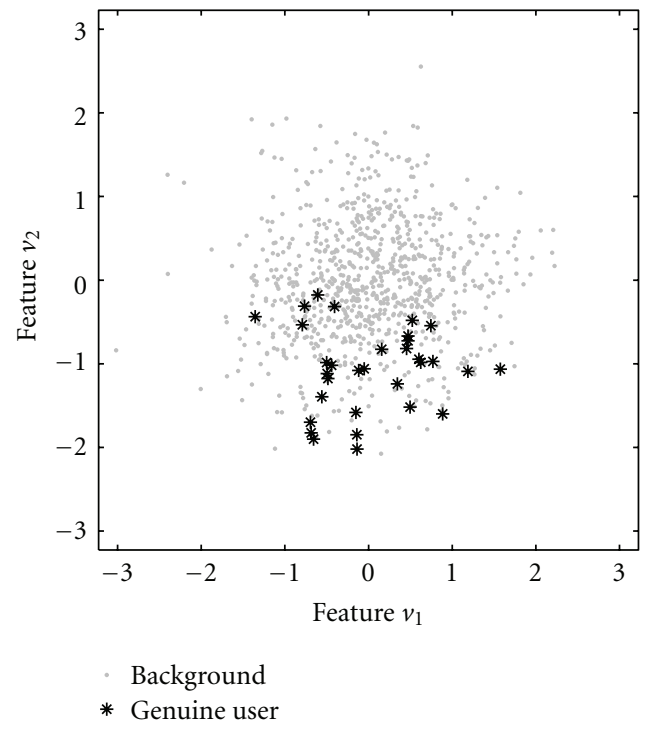

(a)

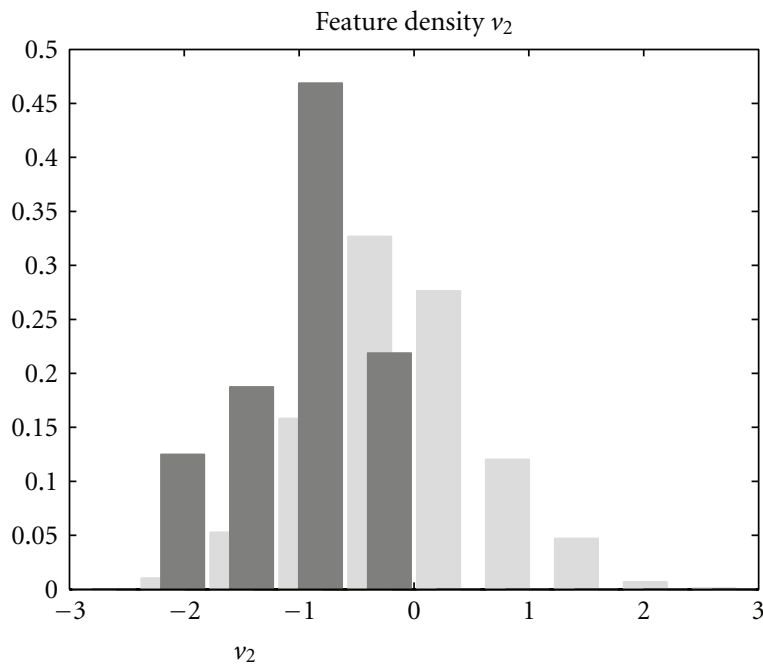

Background

Genuine user

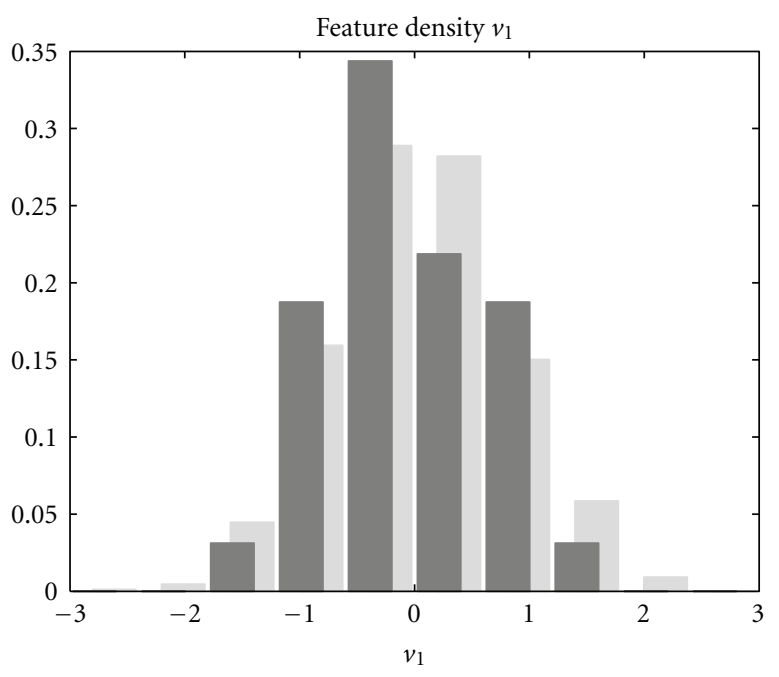

Background

Genuine user

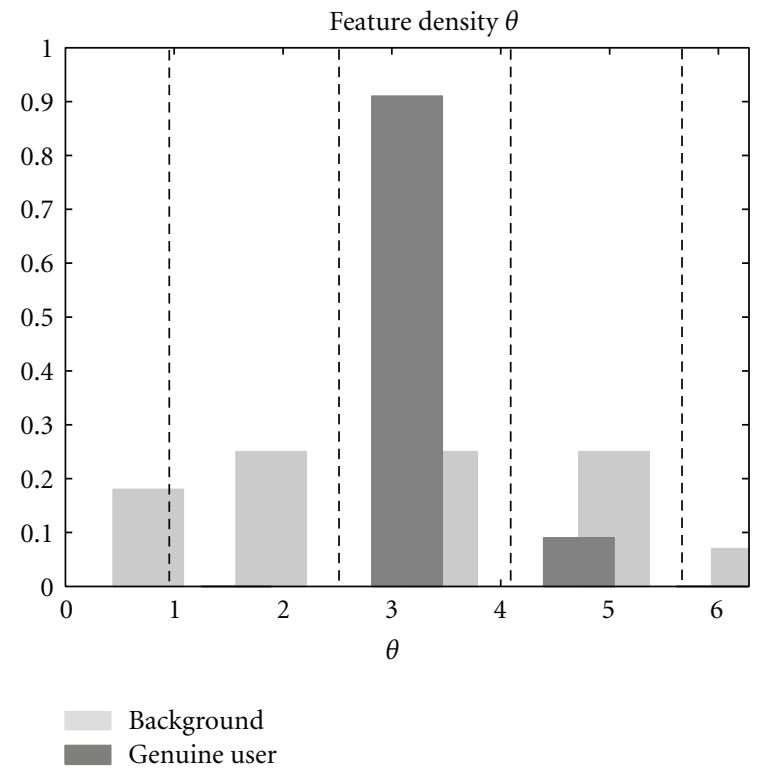

(d)

FIGURE 18: An example of the feature density based on LS pairing and APQ. (a) The two-dimensional feature density; (b) the density of $v_{1}$; (c) the density of $v_{2}$; (d) the pairwise phase density of $\left\{v_{1} v_{2}\right\}$, with the adaptive quantization boundaries (dashed line).

"size" densities and moderate $\bar{d}$ values. Thus it avoids small $\bar{d}$ values and effectively maximizes (16).

4.5. Verification Performance. We test the performances of $\mathrm{LS}+\mathrm{APQ}$ at various numbers of input features $D$ as well as various numbers of quantization bits $b \in\{1, \ldots, 6\}$. The performances are further compared with the onedimensional fixed quantization (1D FQ) [17]. The EER results for FVC2000 and FRGC are shown in Table 3 and Figure 15.
Both data sets show that by increasing the number of features $D$ at a fixed $b$-bit quantization per feature pair, the performances of LS + APQ improves and becomes stable. Additionally, given $D$ features, the overall performances of $\mathrm{LS}+\mathrm{APQ}$ are relatively good only when $b \leq 3$. However, when $b \geq 4$, the performances become poor. For FVC2000, an average of 1-bit per feature pair gives the lowest EER, while for FRGC, the lowest EER allows 2-bit per feature pair. In Figure 16, we give their FAR/FRR performances at the best $D$, with $b$ from 1 to 4 , and the FAR/FRR performances at the best $b$ are given in Table 4 . 
TABLE 3: The EER performances of LS + APQ and ID FQ, at various feature dimensionality $D$ and various numbers of quantization bits $b$, for (a) FVC2000 and (b) FRGC.

(a)

\begin{tabular}{|c|c|c|c|c|c|c|c|}
\hline \multicolumn{2}{|c|}{ FVC2000 } & \multicolumn{6}{|c|}{$D_{\mathrm{PCA}}=D, \mathrm{EER}=(\%)$} \\
\hline & & $D=50$ & 100 & 150 & 200 & 250 & 300 \\
\hline \multirow{6}{*}{$\mathrm{LS}+\mathrm{APQ}$} & $b=1$ & 4.4 & 2.8 & 2.0 & 1.9 & 1.8 & 1.9 \\
\hline & $b=2$ & 4.6 & 3.0 & 2.0 & 2.1 & 1.7 & 1.6 \\
\hline & $b=3$ & 6.4 & 3.7 & 2.8 & 2.6 & 2.5 & 2.7 \\
\hline & $b=4$ & 8.2 & 5.9 & 4.6 & 3.4 & 3.2 & 3.3 \\
\hline & $b=5$ & 10.0 & 6.6 & 5.9 & 4.4 & 4.0 & 3.7 \\
\hline & $b=6$ & 11.4 & 7.1 & 6.6 & 5.4 & 4.7 & 4.7 \\
\hline \multirow{3}{*}{ 1D FQ } & $b=1$ & 6.7 & 4.0 & 2.9 & 2.6 & 2.7 & 2.3 \\
\hline & $b=2$ & 7.5 & 5.3 & 4.2 & 3.6 & 3.6 & 3.6 \\
\hline & $b=3$ & 9.2 & 6.4 & 5.5 & 5.0 & 5.2 & 4.9 \\
\hline
\end{tabular}

(b)

\begin{tabular}{|c|c|c|c|c|c|c|c|c|}
\hline \multicolumn{2}{|c|}{ FRGC } & \multicolumn{7}{|c|}{$D_{\mathrm{PCA}}=500, D_{\mathrm{LDA}}=D, \mathrm{EER}=(\%)$} \\
\hline & & $D=50$ & 80 & 100 & 120 & 150 & 180 & 200 \\
\hline \multirow{6}{*}{$\mathrm{LS}+\mathrm{APQ}$} & $b=1$ & 4.0 & 3.4 & 3.0 & 2.6 & 2.9 & 2.7 & 2.7 \\
\hline & $b=2$ & 3.5 & 3.0 & 2.8 & 2.3 & 2.8 & 2.7 & 2.9 \\
\hline & $b=3$ & 4.7 & 4.1 & 3.7 & 3.4 & 3.3 & 3.6 & 3.9 \\
\hline & $b=4$ & 6.7 & 5.9 & 5.0 & 4.8 & 4.7 & 5.0 & 5.2 \\
\hline & $b=5$ & 8.1 & 7.0 & 6.3 & 6.1 & 6.5 & 6.6 & 6.4 \\
\hline & $b=6$ & 10.1 & 8.6 & 7.5 & 7.2 & 7.2 & 7.4 & 7.6 \\
\hline \multirow{3}{*}{ 1D FQ } & $b=1$ & 5.7 & 4.7 & 4.2 & 4.0 & 4.1 & 4.1 & 4.2 \\
\hline & $b=2$ & 5.1 & 5.4 & 5.1 & 5.0 & 5.2 & 5.9 & 6.1 \\
\hline & $b=3$ & 6.5 & 6.5 & 6.4 & 6.2 & 6.5 & 6.9 & 7.3 \\
\hline
\end{tabular}

TABLE 4: The FAR/FRR performances for FVC2000 and FRGC at the best $D$ - $L$ setting.

\begin{tabular}{lccc}
\hline FRR $(\%)$ & FAR $=10^{-4}$ & $10^{-3}$ & $10^{-2}$ \\
\hline FVC2000, $D=300, L=300$ & 17.2 & 9.6 & 2.6 \\
FRGC, $D=120, L=120$ & 14.7 & 8.2 & 3.7 \\
\hline
\end{tabular}

We further compare the LS + APQ with the 1D FQ. In order to compare at the same string length, we compare the $b / 2$-bit $1 \mathrm{D}$ FQ with the $b$-bit $\mathrm{LS}+\mathrm{APQ}$. The EER performances in Figure 15 show that in general when $b \leq 3$, $\mathrm{LS}+\mathrm{APQ}$ outperforms $1 \mathrm{D}$ FQ. However, when $b \geq 4$, LS + APQ is no longer competitive to $1 \mathrm{D}$ FQ. In Figure 17, we give an example of comparing the FAR/FRR performances of LS + APQ and 1D FQ, on FRGC. Since both APQ and FQ provide equal-probability intervals, they yield almost the same FAR performance. On the other hand, LS + APQ obtains lower FRR as compared with 1D FQ.

In [19], it was shown that FQ in combination with the DROBA adaptive bit allocation principle (FQ + DROBA) provides considerably good performances. Therefore, we compare the LS + APQ with the FQ + DROBA. In order to compare both methods at the same $D$ - $L$ setting, for LS
TABLE 5: The EER performances of LS + APQ and FQ + DROBA, at at several $D-L$ settings, for (a) FVC2000 and (b) FRGC.

(a)

\begin{tabular}{lccc}
\hline FVC2000 & \multicolumn{3}{c}{$D=250$, EER $=(\%)$} \\
& $L=50$ & $L=100$ & $L=150$ \\
\hline LS + APQ & 2.3 & 1.7 & 1.9 \\
FQ + DROBA & 2.4 & 2.1 & 2.2 \\
\hline
\end{tabular}

(b)

\begin{tabular}{lccc}
\hline FRGC & \multicolumn{3}{c}{$D=120$, EER $=(\%)$} \\
& $L=60$ & $L=90$ & $L=120$ \\
\hline LS + APQ & 2.3 & 2.4 & 2.3 \\
FQ + DROBA & 2.4 & 2.6 & 2.8 \\
\hline
\end{tabular}

$+\mathrm{APQ}$, we extract only $2 K$ features from the $D$ features, thus $K$ pairs from the LS pairing. Afterwards, we apply the 2-bit APQ for every feature pair (see Figure 3 ). In this case, $K=L / 2$. Table 5 shows the EER performances of LS + APQ and FQ + DROBA at several different $D$ - $L$ settings. Results show that LS + APQ obtains slightly better performances than FQ + DROBA. 


\section{Discussion}

Essentially, the pairwise phase quantization involves two user-specific adaptation steps: the long-short (LS) pairing, as well as the adaptive phase quantization (APQ). From the pairing's perspective, although we only quantize the phase, the magnitude information (i.e. the feature mean) is not discarded. Instead, it is employed in the LS pairing strategy to facilitate extracting distinctive phase bits. Additionally, although with low computational complexity, the LS pairing strategy is effective for arbitrary feature densities. From the quantizer's perspective, quantizing in phase domain has the advantage that a circularly symmetric two-dimensional feature density results in a simple uniform phase density. Additionally, we apply user-specific phase adaptation. As a result, the extracted phase bits are not only distinctive but also robust to over-fitting. However, the experimental results imply that such advantages only exist when $b \leq 3$. To summarize, as illustrated in Figure 18, the LS pairing is a user-specific resampling procedure that provides simple unform but distinctive phase densities. The APQ further enhances the feature distinctiveness by adjusting the userspecific phase quantization intervals.

\section{Conclusion}

Extracting binary biometric strings is a fundamental step in biometric compression and template protection. Unlike many previous work which quantize features individually, in this paper, we propose a pairwise adaptive phase quantization (APQ), together with a long-short (LS) pairing strategy, which aims to maximize the overall detection rate. Experimental results on the FVC2000 and the FRGC database show reasonably good verification performances.

\section{Acknowledgment}

This research is supported by the research program Sentinels (http://www.sentinels.nl/). Sentinels is being financed by Technology Foundation STW, the Netherlands Organization for Scientific Research (NWO), and the Dutch Ministry of Economic Affairs.

\section{References}

[1] A. K. Jain, K. Nandakumar, and A. Nagar, "Biometric template security," EURASIP Journal on Advances in Signal Processing, vol. 2008, Article ID 579416, 2008.

[2] N. K. Ratha, J. H. Connell, and R. M. Bolle, "Enhancing security and privacy in biometrics-based authentication systems," IBM Systems Journal, vol. 40, no. 3, pp. 614-634, 2001.

[3] N. K. Ratha, S. Chikkerur, J. H. Connell, and R. M. Bolle, "Generating cancelable fingerprint templates," IEEE Transactions on Pattern Analysis and Machine Intelligence, vol. 29, no. 4, pp. 561-572, 2007.

[4] A. Juels and M. Sudan, "A fuzzy vault scheme," Designs, Codes, and Cryptography, vol. 38, no. 2, pp. 237-257, 2006.

[5] K. Nandakumar, A. K. Jain, and S. Pankanti, "Fingerprintbased fuzzy vault: implementation and performance," IEEE
Transactions on Information Forensics and Security, vol. 2, no. 4, pp. 744-757, 2007.

[6] A. Juels and M. Wattenberg, "Fuzzy commitment scheme," in Proceedings of the 6th ACM Conference on Computer and Communications Security (ACM CCS '99), pp. 28-36, November 1999.

[7] Y. Dodis, L. Reyzin, and A. Smith, "Fuzzy extractors: how to generate strong keys from biometrics and other noisy data," in Proceedings of International Conference on the Theory and Applications of Cryptographic Techniques, vol. 3027 of Lecture Notes in Computer Science, pp. 523-540, May 2004.

[8] E. C. Chang and S. Roy, "Robust extraction of secret bits from minutiae," in Proceedings of the 2nd International Conference on Biometrics (ICB '07), vol. 4642 of Lecture Notes in Computer Science, pp. 750-759, 2007.

[9] J.-P. Linnartz and P. Tuyls, "New shielding functions to enhance privacy and prevent misuse of biometrie templates," in Proceedings of Audio-and Video-Based Biometrie Person Authentication (AVBPA '03), vol. 2688 of Lecture Notes in Computer Science, pp. 393-402, Guildford, UK, 2003.

[10] P. Tuyls, A. H. M. Akkermans, T. A. M. Kevenaar, G.-J. Schrijen, A. M. Bazen, and R. N. J. Veldhuis, "Practical biometric authentication with template protection," in Proceedings of the 5th International Conference on Audio-and Video-Based Biometric Person Authentication (AVBPA '05), vol. 3546 of Lecture Notes in Computer Science, pp. 436-446, Hilton Rye Town, NY, USA, July 2005.

[11] T. A. M. Kevenaar, G. J. Schrijen, M. van der Veen, A. H. M. Akkermans, and F. Zuo, "Face recognition with renewable and privacy preserving binary templates," in Proceedings of the 4th IEEE Workshop on Automatic Identification Advanced Technologies (AUTO ID '05), pp. 21-26, New York, NY, USA, October 2005.

[12] F. Hao, R. Anderson, and J. Daugman, "Combining crypto with biometrics effectively," IEEE Transactions on Computers, vol. 55, no. 9, pp. 1081-1088, 2006.

[13] A. B. J. Teoh, A. Goh, and D. C. L. Ngo, "Random multispace quantization as an analytic mechanism for BioHashing of biometric and random identity inputs," IEEE Transactions on Pattern Analysis and Machine Intelligence, vol. 28, no. 12, pp. 1882-1901, 2006.

[14] C. Vielhauer, R. Steinmetz, and A. Mayerhöfer, "Biometric hash based on statistical features of online signatures," in Proceedings of the 16th International Conference on Pattern Recognition (ICPR '02), vol. 1, pp. 123-126, Quebec, Canada, 2002.

[15] H. Feng and C. C. Wah, "Private key generation from online handwritten signatures," Information Management and Computer Security, vol. 10, no. 4, pp. 159-164, 2002.

[16] Y. -J. Chang, W. Zhang, and T. Chen, "Biometrics-based cryptographic key generation," in Proceedings of the IEEE International Conference on Multimedia and Expo (ICME '01), vol. 3, pp. 2203-2206, Taipei, Taiwan, June 2004.

[17] C. Chen, R. N. J. Veldhuis, T. A. M. Kevenaar, and A. H. M. Akkermans, "Multi-bits biometric string generation based on the likelihood ratio," in Proceedings of the 1st IEEE International Conference on Biometrics: Theory, Applications, and Systems (BTAS '07), September 2007.

[18] C. Chen, R. N. J. Veldhuis, T. A. M. Kevenaar, and A. H. M. Akkermans, "Biometric binary string generation with detection mate optimized bit allocation," in Proceedings of the IEEE Computer Society Conference on Computer Vision and Pattern Recognition Workshops (CVPR '08), June 2008. 
[19] C. Chen, R. N. J. Veldhuis, T. A. M. Kevenaar, and A. H. M. Akkermans, "Biometric quantization through detection rate optimized bit allocation," EURASIP Journal on Advances in Signal Processing, vol. 2009, Article ID 784834, 2009.

[20] C. Chen and R. N. J. Veldhuis, "Extracting biometric binary strings with minimal area under the frr curve for the hamming distance classifier," in Proceedings of the 17th European Signal Processing Conference (EUSIPCO '09), 2009.

[21] C. Chen and R. Veldhuis, "Binary biometric representation through pairwise polar quantization," in Proceedings of the $3 \mathrm{rd}$ International Conference on Advances in Biometrics (ICB '09), vol. 5558 of Lecture Notes in Computer Science, pp. 72-81, Alghero, Italy, June 2009.

[22] J. Daugman, "The importance of being random: statistical principles of iris recognition," Pattern Recognition, vol. 36, no. 2, pp. 279-291, 2003.

[23] D. Maio, D. Maltoni, R. Cappelli, J. L. Wayman, and A. K. Jain, "FVC2000: fingerprint verification competition," IEEE Transactions on Pattern Analysis and Machine Intelligence, vol. 24, no. 3, pp. 402-412, 2002.

[24] P. J. Phillips, P. J. Flynn, T. Scruggs et al., "Overview of the face recognition grand challenge," in Proceedings of IEEE Computer Society Conference on Computer Vision and Pattern Recognition (CVPR '05), pp. 947-954, San Diego, Calif, USA, June 2005.

[25] R. Veldhuis, A. Bazen, J. Kauffman, and P. Hartel, "Biometric verification based on grip-pattern recognition," in Security, Steganography, and Watermaking of Multimedia Contents VI, vol. 5306 of Proceedings of SPIE, pp. 634-641, San Jose, Calif, USA, January 2004.

[26] M. Gardner, The Binary Gray Code, W. H. Freeman, New York, NY, USA, 1986. 


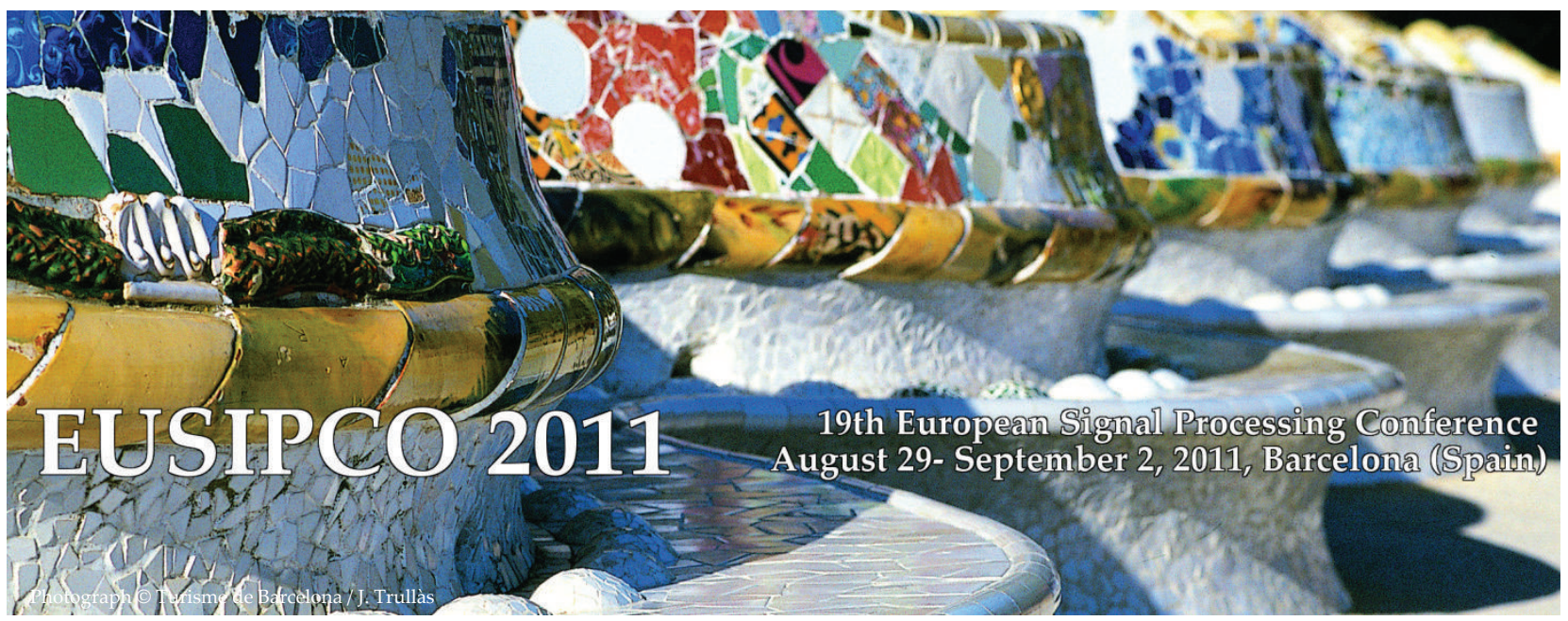

\section{Preliminary call for papers}

The 2011 European Signal Processing Conference (EUSIPCO-2011) is the nineteenth in a series of conferences promoted by the European Association for Signal Processing (EURASIP, www.eurasip.org). This year edition will take place in Barcelona, capital city of Catalonia (Spain), and will be jointly organized by the Centre Tecnològic de Telecomunicacions de Catalunya (CTTC) and the Universitat Politècnica de Catalunya (UPC).

EUSIPCO-2011 will focus on key aspects of signal processing theory and applications as listed below. Acceptance of submissions will be based on quality, relevance and originality. Accepted papers will be published in the EUSIPCO proceedings and presented during the conference. Paper submissions, proposals for tutorials and proposals for special sessions are invited in, but not limited to, the following areas of interest.

\section{Areas of Interest}

- Audio and electro-acoustics.

- Design, implementation, and applications of signal processing systems.

- Multimedia signal processing and coding.

- Image and multidimensional signal processing.

- Signal detection and estimation.

- Sensor array and multi-channel signal processing.

- Sensor fusion in networked systems.

- Signal processing for communications.

- Medical imaging and image analysis.

- Non-stationary, non-linear and non-Gaussian signal processing.

\section{Submissions}

Procedures to submit a paper and proposals for special sessions and tutorials will be detailed at www.eusipco2011.org. Submitted papers must be camera-ready, no more than 5 pages long, and conforming to the standard specified on the EUSIPCO 2011 web site. First authors who are registered students can participate in the best student paper competition.

\section{Important Deadlines:}

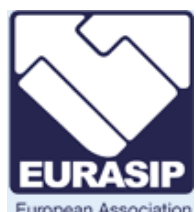

for Signal Procesing
Proposals for special sessions

Proposals for tutorials

Electronic submission of full papers

Notification of acceptance

Submission of camera-ready papers
15 Dec 2010

21 Feb 2011

23 May 2011

6 Jun 2011
18 Feb 2011
Organizing Committee

Honorary Chair

Miguel A. Lagunas (CTTC)

General Chair

Ana I. Pérez-Neira (UPC)

General Vice-Chair

Carles Antón-Haro (CTTC)

Technical Program Chair

Xavier Mestre (CTTC)

Technical Program Co-Chairs

Javier Hernando (UPC)

Montserrat Pardàs (UPC)

Plenary Talks

Ferran Marqués (UPC)

Yonina Eldar (Technion)

Special Sessions

Ignacio Santamaría (Unversidad

de Cantabria)

Mats Bengtsson (KTH)

Finances

Montserrat Nájar (UPC)

Tutorials

Daniel P. Palomar

(Hong Kong UST)

Beatrice Pesquet-Popescu (ENST)

Publicity

Stephan Pfletschinger (CTTC)

Mònica Navarro (CTTC)

Publications

Antonio Pascual (UPC)

Carles Fernández (CTTC)

Industrial Liaison $\mathcal{E}$ Exhibits

Angeliki Alexiou

(University of Piraeus)

Albert Sitjà (CTTC)

International Liaison

Ju Liu (Shandong University-China) Jinhong Yuan (UNSW-Australia)

Tamas Sziranyi (SZTAKI -Hungary) Rich Stern (CMU-USA)

Ricardo L. de Queiroz (UNB-Brazil)

Webpage: www.eusipco2011.org 